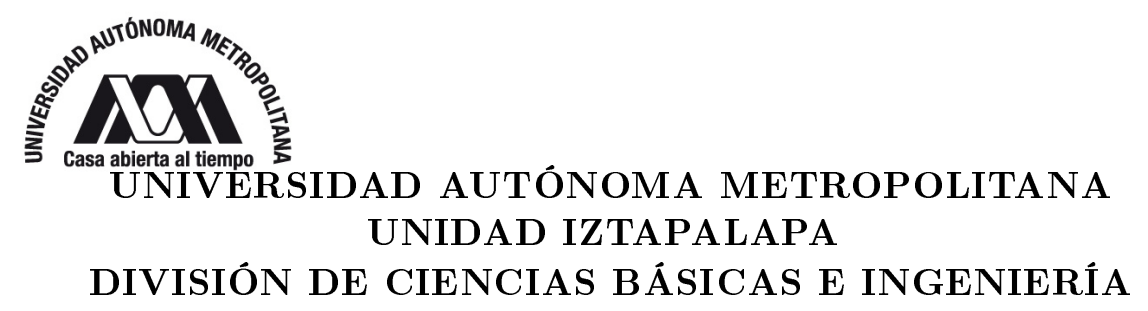

\title{
LA ECUACIÓN DE BALANCE DE MOMENTO \\ ELECTROMAGNÉTICO Y LA DENSIDAD DE FUERZA PARA SÓLIDOS
}

\author{
Tesis que presenta \\ Oscar Daniel Nuñez Valencia \\ Para obtener el grado de: \\ Maestro en Ciencias (Física) \\ Asesor: \\ M. en C. José Luis Jiménez Ramírez \\ Jurado calificador: \\ Presidente: Dr. Victor David Granados García \\ Secretario: M. en C. José Luis Jiménez Ramírez \\ Vocal: Dr. Alberto Rubio Ponce
}

Octubre 20, 2017. Ciudad de México. 

Dedicado a mi hija Daniell Alessandra Nuñez González mi familia 
0.0 . 


\section{Agradecimientos}

Es una oportunidad inmejorable para agradecer a la vida que me ha esperado tanto y a todas las personas que de alguna u otra forma han estado involucrados en este proceso de mi vida.

Agradezco principalmente a mi profesor José Luis Jiménez, por su tiempo, sus consejos y toda la energía vertida en esté trabajo, ademas de los cursos que tuve la fortuna de aprender de él.

A los miembros del Jurado calificador, Dr. Víctor David Granados García y Dr. Alberto Rubio Ponce, por haber leído este trabajo, por sus comentarios y valiosas recomendaciones que surgieron después del análisis del mismo y que complementaron de gran forma este trabajo.

Es evidente que nadie hace las cosas solo en la vida, y aquí quiero señalar lo evidente, agradezco profundamente a mis compañeros de generación César Rosiles, Fernando García, Belen Carvente, Eric Escobar, José Morales y un agradecimiento muy especial a mis amigos y compañeros Elizabeth Cruz y Salvador González, innumerables fueron los días que pasamos hasta tarde en la biblioteca, unos con mas energías, otros con menos, pero siempre con mucha decisión, además valoro las personas que son y el apoyo que recibí de ustedes.

Agradezco especialmente a Sandra Verónica González Lamas, por todo el apoyo físico y moral durante todo este proceso, y por todo el esfuerzo y amor que ha puesto en la vida de nuestra hija, el ver a Alessandra tan amada, me permitió a mi, estar tranquilo para volcar todos mis esfuerzos en este trabajo, el cual hoy ha tomado forma.

Al Consejo Nacional de Ciencia y Tecnología, CONACYT, por el apoyo brindado, no solo a mi, sino a todos los estudiantes de la generación. 
0.0 . 


\section{Resumen}

El tema de la densidad de fuerza en medios materiales surge hace poco más de un centenar de años, y a lo largo de todo este periodo diversos autores han abordado este problema compartiendo una característica en común. Todos los autores consideran que para deducir la densidad de fuerza en medios se debe considerar por separado fluidos y sólidos, además, por supuesto, de distinguir si esta fuerza es debida a campos electrostáticos o magnetostáticos. A pesar de los trabajos que han surgido en este lapso de tiempo este no es un tema agotado. En este trabajo presentamos un método novedoso para calcular la densidad de fuerza en sólidos, este nuevo método está sustentado por una estructura conceptual distinta, puesto que basta con la ecuación de balance de momento electromagnética obtenida directamente de las ecuaciones de Maxwell y las ecuaciones constitutivas para obtener dicha densidad de fuerza, además con esto mostramos la relación directa que tienen las densidades de fuerza en fluidos y sólidos conocidas y la ecuación de balance de momento electromagnético en medios materiales. Esta ecuación aún encierra sorpresas, baste mencionar que ha sido objeto de una controversia por más de 100 años, aunque no es de nuestro interés en este trabajo discutir esta controversia. Con este nuevo método mostramos cómo es posible considerar a ambos medios, fluidos y sólidos en una sola deducción, lo cual es un trabajo que no se ha hecho hasta el momento, proponemos una densidad de fuerza en medios generalizada la cual incluye como casos particulares a las densidades de fuerza usualmente conocidas. 
0.0 .

RESUMEN 


\section{Índice general}

Agradecimientos ..................

Resumen . . . . . . . . . . . . . . . . . . VII

1. Introducción 1

2. Balance de momento electromagnético en el vacío 5

3. Ecuación de Balance de Momento en el Vacío. Un método alterno 9

4. Balance de momento electromagnético en medios materiales 11

4.1. Balance de momento electrostático en medios materiales fluidos. La densidad de fuerza de Helmholtz . . . . . . . . . . . . . . 11

4.2. Balance de momento electromagnético en medios materiales sólidos . . 15

4.3. Densidad de fuerza en campos magnetostáticos. Método de Stratton . . 18

4.4. Deducción de la densidad de fuerza de Helmholtz como un paso particular de la densidad de fuerza en un sólido . . . . . . . . . . . . 23

5. Balance de momento electromagnético en medios materiales. Deducción alternativa

5.1. Balance de momento electromagnético en medios materiales fluidos.

La densidad de fuerza de Helmholtz . . . . . . . . . . . . . 27

5.1.1. Deducción alternativa. Primer método . . . . . . . . . . 28

5.1.2. Deducción alternativa. Segundo método . . . . . . . . . . . 29

5.2. Balance de momento electromagnético en medios materiales sólidos, deducciones alternativas . . . . . . . . . . . . . . 30

5.2.1. Deducción alternativa. Primer método . . . . . . . . . . . 30

5.2.2. Deducción alternativa. Segundo método. . . . . . . . . . . 34

5.3. Densidad de fuerza magnética en medios materiales . . . . . . . . 37

5.3.1. Densidad de fuerza magnética en fluidos . . . . . . . . . 38

5.3.2. Densidad de fuerza magnética en sólidos . . . . . . . . . . 40

5.4. La densidad de fuerza en medios materiales . . . . . . . . . . . . . 42

6. Equivalencia de las diferentes propuestas para el tensor de esfuerzos en un sólido elástico

6.1. Equivalencia de las propuestas para el tensor de esfuerzos de Landau-

Lifshitz y Robinson . . . . . . . . . . . . . . . . 46 
6.2. Equivalencia de las propuestas para el tensor de esfuerzos de Stratton y Landau- Lifshitz . . . . . . . . . . . . . . . . . 46

6.3. Demostración de que en el tensor de Maxwell están incluidas las propuestas de Stratton, Landau- Lifshitz y Robinson . . . . . . . . . . 48

$\begin{array}{ll}\text { 7. Conclusiones } & 51\end{array}$

$\begin{array}{ll}\text { Apéndices } & 54\end{array}$

$\begin{array}{ll}\text { A. Elasticidad } & \mathbf{5 7}\end{array}$

A.1. Descripción material y descripción espacial . . . . . . . . . . 57

A.2. Derivada material . . . . . . . . . . . . . 57

A.3. Tensor de deformación ...................... 58

B. Deducción de la ecuación (4.33) 61

C. Deducción de las ecuaciones (5.10) y (5.11) 63

$\begin{array}{ll}\text { D. Coeficientes del tensor } \alpha_{j k l m} & 65\end{array}$ 


\section{Capítulo 1}

\section{Introducción}

En la literatura de la teoría electromagnética las ecuaciones de balance de momento electromagnético tienen un papel conceptual importante, los métodos usuales para deducir estos balances consisten en partir de la densidad de fuerza del sistema para posteriormente realizar el balance, cuando no se tiene una densidad fuerza, se deduce esta a partir de la energía libre del sistema. A pesar de que este tema ha sido tratado por una gran cantidad de autores, como veremos más adelante, los métodos usados para deducir estas ecuaciones presentan complicaciones que no suelen reconocerse pero que son importantes de señalar.

En el vacío, el camino usual para obtener la ecuación de balance de momento electromagnético es el seguido por Jackson [7], el cual consiste en partir de la segunda ley de Newton y considerar que la fuerza sobre el sistema es la densidad de fuerza de Lorentz, en esta densidad de fuerza aparecen densidades de carga y corriente, las cuales se toman de las ecuaciones de Maxwell, después se usa cálculo vectorial y tensorial y se obtiene la ecuación de balance de momento en el vacío. Por otra parte en medios materiales el problema de deducir la ecuación de balance de momento electromagnético es más complicado ya que no se cuenta con el análogo a la densidad de fuerza de Lorentz, por ello, usualmente, se tiene que deducir primero una densidad de fuerza. Para analizar la densidad de fuerza en medios materiales, usualmente se consideran dos casos, como menciona Landau y Lifshitz [10] "El problema de calcular las fuerzas (llamadas a veces fuerzas ponderomotrices) que actúan sobre los dieléctricos en un campo eléctrico no homogéneo arbitrario, es bastante complicado y exige considerar separadamente los líquidos (o gases) y los sólidos". Este es el camino que se ha seguido usualmente, no solo por Landau y Lifshitz [10] sino también por autores como Stratton [1] y Robinson [4], entre otros, todos comparten la idea de considerar por separado estos medios materiales.

La manera habitual de deducir la densidad de fuerza cuando el medio es un fluido y considerando únicamente campos electrostáticos, es la seguida por Helmholtz, quien en 1882 usó el principio de trabajo virtual, la energía libre del sistema y las ecuaciones de Maxwell macroscópicas para deducir una densidad de fuerza, conocida actualmente como la densidad de fuerza de Helmholtz, $\vec{f}_{H}$. Después de deducir esta densidad de fuerza, Helmholtz no continuó con la deducción de la ecuación de balance de momento 
explícitamente, solo postuló un tensor a partir del cual se puede obtener esta densidad de fuerza. Posteriormente a Helmholtz diversos autores como Stratton [1], Panofsky y Phillips [2], Robinson [4], Tamm [9], Landau y Lifshitz [10], entre otros, han abordado este problema, unos con más hipótesis que otros. La característica en común que comparten estos autores es que todos siguen el mismo método, teniendo la misma estructura conceptual del problema, es decir, primero deducir una densidad de fuerza y posteriormente obtener el balance de momento.

Pese a que la deducción de la densidad de fuerza en medios es un problema puramente macroscópico, como bien lo hace notar Robinson [6], diversos autores como Gordon [5], Peierls [8], Lai et al [11] y [12] entre otros, han hecho deducciones microscópicas para obtener esta densidad de fuerza, lo cual deja aún más claro el interés por la densidad de fuerza en medios.

Cuando el medio es un sólido elástico se deduce la densidad de fuerza adecuada pero, a diferencia de cuando se analiza el problema en el vacío y en los fluidos, usualmente no se suele proceder a deducir la ecuación de balance de momento salvo algunas excepciones como lo es Robinson [4], dejando así por muchos años incompleto este problema.

Si bien los temas de las densidades de fuerza sobre los medios materiales fluidos o sólidos debidas a campos electrostáticos son abordados en libros y artículos, cuando se trata de analizar las densidades de fuerza debidas a campos magnetostáticos, el análisis es escaso. Usualmente la deducción de la densidad de fuerza debida a campos magnetostáticos se presenta como una analogía a la de la densidad de fuerza debida a campos electrostáticos. La deducción se basa en partir de la densidad de energía libre del sistema, después se procede a realizar su variación y usando la ecuación constitutiva que vincula los campos $\vec{B}$ y $\vec{H}$, se deduce la expresión para la densidad de fuerza, a la cual llamaremos solamente, densidad de fuerza magnetostática. Cuando el medio es un fluido también existen deducciones microscópicas de esta densidad de fuerza hechas por Campos et al [15].

Hasta ahora hemos hecho énfasis que en los métodos usuales para deducir las densidades de fuerza, suelen partir de la expresión usual para la energía libre en el medio, hacemos hincapié en esto porque consideramos que este problema se puede resolver sin necesidad de partir de la energía libre para el sistema, además, considerando el poco análisis de la densidad de fuerza magnetostática, nos sugiere analizar este problema desde otra perspectiva.

Motivados por el problema de deducir la densidad de fuerza en medios materiales, presentamos un nuevo método para deducir esta densidad de fuerza. A diferencia del método usual, este nuevo método no solo presenta un camino alterno para deducir la ecuación de balance de momento electromagnético, sino, más bien, una estructura conceptual distinta. Esta nueva estructura conceptual surge al considerar que este problema puede analizarse mediante las ecuaciones de Maxwell y las relaciones constitutivas, puesto que en las relaciones constitutivas se encuentran las características del medio, y como responde éste a los campos, es decir debe bastar con ellas para analizar el problema de interacción campos electromagnéticos-materia.

Así que, partiendo directamente de las ecuaciones de Maxwell y con ayuda del 
cálculo vectorial y tensorial, deducimos una ecuación de balance de momento, sin necesidad de deducir previamente una densidad de fuerza como hizo Helmholtz y los autores antes mencionados. Una vez obtenida esta ecuación de balance de momento obtenemos la densidad de fuerza. La densidad de fuerza que surge a partir de este balance luce como una densidad de fuerza general, es decir, en principio podría describir a un fluido o un sólido, puesto que para obtener esta densidad de fuerza no se han hecho hipótesis que limiten su generalidad.

Con el fin de mostrar que tan general es esta ecuación de balance de momento, analizamos la densidad de fuerza que contiene. Nuestro objetivo principal es mostrar que la ecuación de balance de momento electromagnético, obtenida directamente de las ecuaciones de Maxwell, contiene ya las densidades de fuerza electrostáticas y magnetostáticas para fluidos y sólidos, esto es algo que no se ha realizado previamente y con lo cual pretendemos resolver el problema de las densidades de fuerza en medios materiales.

Hasta ahora se ha mostrado parcialmente que la densidad de fuerza de Helmholtz está contenida en la ecuación de balance, interpretada como lo hacemos aquí, pero aún no se ha mostrado si la densidad de fuerza en un sólido está también contenida en esta ecuación de balance, esto es precisamente el objetivo principal de este trabajo, para así exhibir la generalidad de la ecuación de balance así como la coherencia de esta interpretación y lo que ella ofrece.

$\mathrm{Al}$ exhibir que la ecuación de balance, así interpretada, contiene a la densidad de fuerza de un sólido, damos un elemento más para mostrar el alcance de la ecuación de balance de momento electromagnético que se deduce directamente de las ecuaciones de Maxwell, además, con esto hemos resuelto el problema de la deducción de la densidad de fuerza de una manera general, al menos para fluidos y sólidos, pues ambas densidades de fuerza están contenidas en una sola ecuación, es decir tenemos una estructura general para estos dos casos, a diferencia de lo mencionado por Landau y Lifshitz quienes mencionan que estos dos casos se deben tratar por separado. Por otra parte al mostrar que las densidades de fuerza para un sólido y para un fluido están contenidas en esta nueva ecuación de balance de momento, tenemos un punto más a favor de nuestra estructura conceptual, la cual considera que la interacción campos electromagnéticos con sólidos o fluidos puede describirse mediante las ecuaciones de Maxwell y las relaciones constitutivas. 
1.0 . 


\section{Capítulo 2}

\section{Balance de momento electromagnético en el vacío}

En el vacío, el método más usual para obtener la ecuación de balance de momento electromagnético es el seguido por Jackson, este método a pesar de ser aceptado es un método inconsistente. Para mostrar estas inconsistencias es necesario detallar los pasos seguidos por Jackson, aclarando que es de nuestro interés el señalar donde surgen dichas inconsistencias para así facilitar el análisis. Consideramos que analizar la ecuación de balance de momento electromagnético en el vacío tiene importancia, puesto que es un punto de partida para mostrar los problemas conceptuales que se enfrentan al deducir las ecuaciones de balance de momento

Jackson parte de la segunda ley de Newton

$$
\frac{d \vec{P}_{m e c}}{d t}=\vec{F},
$$

y posteriormente considera que $\vec{F}$ es la fuerza electromagnética total sobre una partícula, es decir la fuerza de Lorentz, que como es sabido depende solo de campos externos. En este punto surge la primera inconsistencia, puesto que Jackson no aclara que la fuerza $\vec{F}$ depende solo de campos externos. Si hacemos esta distinción en la fuerza debemos escribir $\vec{F}$ como

$$
\vec{F}=\int\left(\rho \vec{E}_{e x t}+\vec{j} \times \vec{B}_{e x t}\right) d V
$$

sin embargo Jackson no tiene cuidado de hacer esta distinción y escribe la fuerza como

$$
\vec{F}=\int(\rho \vec{E}+\vec{j} \times \vec{B}) d V
$$

así que considerando a (2.2) como la fuerza, escribe la ecuación de partida de la siguiente forma

$$
\frac{d \vec{P}_{m e c}}{d t}=\int(\rho \vec{E}+\vec{j} \times \vec{B}) d V
$$


En el siguiente paso sustituye las densidades de carga y corriente $\rho$ y $\vec{j}$ de la fuerza por las que aparecen en las ecuaciones de Maxwell, las cuales dependen de los campos totales, es decir

$$
\rho=\epsilon_{0}\left(\nabla \cdot \vec{E}_{t o t}\right)
$$

y

$$
\vec{j}=\frac{1}{\mu_{0}}\left(\nabla \times \vec{B}_{t o t}-\epsilon_{0} \mu_{0} \frac{\partial \vec{E}_{t o t}}{\partial t}\right) .
$$

En este paso Jackson, una vez más, pasa por alto el señalar que los campos involucrados en las ecuaciones de Maxwell son campos totales, así que omitiendo esta distinción y con ayuda de las ecuaciones (2.4) y (2.5) escribe la siguiente igualdad

$$
\rho \vec{E}+\vec{j} \times \vec{B}=\left[\epsilon_{0}(\nabla \cdot \vec{E}) \vec{E}+\frac{1}{\mu_{0}}\left(\nabla \times \vec{B}-\epsilon_{0} \mu_{0} \frac{\partial \vec{E}}{\partial t}\right) \times \vec{B}\right]
$$

la cual es incorrecta, puesto que, si se hace la distinción apropiada de los campos, esta ecuación debería ser escrita como

$$
\rho \vec{E}_{e x t}+\vec{j} \times \vec{B}_{e x t}=\left[\epsilon_{0}\left(\nabla \cdot \vec{E}_{t o t}\right) \vec{E}_{e x t}+\frac{1}{\mu_{0}}\left(\nabla \times \vec{B}_{t o t}-\epsilon_{0} \mu_{0} \frac{\partial \vec{E}_{t o t}}{\partial t}\right) \times \vec{B}_{e x t}\right]
$$

Aquí surge una segunda inconsistencia, puesto que en este punto es muy importante el hacer o no una distinción entre campos externos y campos totales. Si hacemos la distinción tal como debe de ser, no es posible ya continuar con la deducción del balance de momento. Dejando a un lado la distinción de los campos externos y totales Jackson escribe la igualdad (2.6).

Cabe enfatizar que los pasos siguientes que desembocan en la ecuación de balance de momento electromagnético en el vacío no tienen sentido si se hace una distinción adecuada de los campos. Jackson con el fin de encontrar el balance hace uso de la siguiente igualdad

$$
\vec{B} \times \frac{\partial \vec{E}}{\partial t}=-\frac{\partial}{\partial t}(\vec{E} \times \vec{B})+\vec{E} \times \frac{\partial \vec{B}}{\partial t}
$$

y suma un cero de la siguiente forma

$$
\vec{B}(\nabla \cdot \vec{B})=0,
$$

con lo cual puede escribir la igualdad (2.6) como

$$
\begin{aligned}
& \rho \vec{E}+\vec{j} \times \vec{B}=\epsilon_{0}[\vec{E}(\nabla \cdot \vec{E})-\vec{E} \times(\nabla \times \vec{E})]+ \\
& \frac{1}{\mu_{0}}[\vec{B}(\nabla \cdot \vec{B})-\vec{B} \times(\nabla \times \vec{B})]-\epsilon_{0} \frac{\partial}{\partial t}(\vec{E} \times \vec{B}),
\end{aligned}
$$


con esto puede escribir la ecuación (2.3) de la siguiente forma

$$
\begin{aligned}
& \frac{d \vec{P}_{m e c}}{d t}+\frac{d}{d t} \int \epsilon_{0}(\vec{E} \times \vec{B}) d V= \\
& \quad \int\left\{\epsilon_{0}[\vec{E}(\nabla \cdot \vec{E})-\vec{E} \times(\nabla \times \vec{E})]+\frac{1}{\mu_{0}}[\vec{B}(\nabla \cdot \vec{B})-\vec{B} \times(\nabla \times \vec{B})]\right\} d V .
\end{aligned}
$$

En este punto Jackson denota a la segunda integral del primer miembro como el momento electromagnético total $\vec{P}_{\text {campo }}$ en el volumen V

$$
\vec{P}_{\text {campo }}=\int \epsilon_{0}(\vec{E} \times \vec{B}) d V,
$$

y al integrando como una densidad de momento electromagnético

$$
\vec{g}=\epsilon_{0}(\vec{E} \times \vec{B})
$$

Después de este punto continúa con el análisis de los términos de la ecuación (2.10), donde, después de un cálculo usando componentes obtiene que

$$
[\vec{E}(\nabla \cdot \vec{E})-\vec{E} \times(\nabla \times \vec{E})]_{\alpha}=\sum_{\beta} \frac{\partial}{\partial x_{\beta}}\left(E_{\alpha} E_{\beta}-\frac{1}{2} \vec{E} \cdot \vec{E} \delta_{\alpha \beta}\right),
$$

posteriormente define el tensor de esfuerzos de Maxwell como

$$
T_{\alpha \beta}=\epsilon_{0} E_{\alpha} E_{\beta}+\frac{1}{\mu_{0}} B_{\alpha} B_{\beta}-\frac{1}{2}\left[\epsilon_{0} \vec{E} \cdot \vec{E}+\frac{1}{\mu_{0}} \vec{B} \cdot \vec{B}\right] \delta_{\alpha \beta},
$$

con lo cual puede escribir finalmente la ecuación (2.10) como

$$
\frac{d}{d t}\left(\vec{P}_{m e c}+\vec{P}_{c a m p o}\right)_{\alpha}=\sum_{\beta} \int \frac{\partial}{\partial x_{\beta}} T_{\alpha \beta} d V
$$

la cual es, precisamente, la ecuación de balance de momento electromagnético en el vacío.

Hasta aquí hemos reproducido los pasos seguidos por Jackson [7] para deducir la ecuación de balance de momento. Aún y cuando este método permite deducir la ecuación de balance, es claro ver que en el camino surgen inconsistencias, así que debido a estas inconsistencias es natural el cuestionar dicho método además de pensar en las limitaciones que tendría el tratar de aplicar este método a un medio con otras características. Tomando en cuenta estas complicaciones es necesario buscar una forma alterna de calcular la ecuación de balance en el vacío, y así evitar pasar por las inconsistencias que contiene el método usual. 
2.0 . 


\section{Capítulo 3}

\section{Ecuación de Balance de Momento en el Vacío. Un método alterno}

Entonces podemos darnos cuenta que la forma usual de vincular la densidad de fuerza de Lorentz con las ecuaciones de Maxwell es inconsistente, esto implica un problema conceptual importante puesto que para seguir adelante con este método tendríamos que hacer caso omiso de las diferencias entre campos externos y campos totales, pero si hacemos esto estaríamos aceptando que la fuerza del sistema no es la fuerza de Lorentz y tendríamos que justificar que especie de fuerza es esta, puesto que depende de campos totales.

Una vez señaladas las inconsistencias de este método usual, la cuestión ahora es usar un método alterno para obtener la ecuación de balance en el vacío y así evitar las inconsistencias que el método usual presenta.

Un método alterno es partir directamente de las ecuaciones de Maxwell en el vacío

$$
\begin{gathered}
\nabla \cdot \vec{E}=\frac{\rho}{\epsilon_{0}}, \\
\nabla \cdot \vec{B}=0, \\
\nabla \times \vec{E}=-\frac{\partial \vec{B}}{\partial t}
\end{gathered}
$$

y

$$
\nabla \times \vec{B}=\mu_{0}\left(\vec{J}+\epsilon_{0} \frac{\partial \vec{E}}{\partial t}\right) .
$$

Manipulando las ecuaciones de Maxwell mediante multiplicaciones escalares y vectoriales realizamos la siguientes operacioń $\vec{E}(3.1)+\frac{1}{\mu_{0}} \vec{B}(3.2)-\epsilon_{0} \vec{E} \times(3.3)-\vec{B} \times(3.4)$, lo cual da origen a la siguiente ecuación

$\epsilon_{0} \vec{E}(\nabla \cdot \vec{E})+\frac{1}{\mu_{0}} \vec{B}(\nabla \cdot \vec{B})-\epsilon_{0} \vec{E} \times(\nabla \times \vec{E})-\frac{1}{\mu_{0}} \vec{B} \times(\nabla \times \vec{B})=\rho \vec{E}+\vec{J} \times \vec{B}+\frac{\partial}{\partial t} \epsilon_{0}(\vec{E} \times \vec{B})$ 
3.0 .

Utilizando identidades tensoriales podemos escribir la siguientes igualdades

$$
\vec{E}(\nabla \cdot \vec{E})-\vec{E} \times(\nabla \times \vec{E})=\nabla \cdot[\vec{E} \vec{E}-(1 / 2) \vec{E} \cdot \vec{E}],
$$

y

$$
\vec{B}(\nabla \cdot \vec{B})-\vec{B} \times(\nabla \times \vec{B})=\nabla \cdot[\vec{B} \vec{B}-(1 / 2) \vec{B} \cdot \vec{B}],
$$

donde $\vec{E} \vec{E}$ y $\vec{B} \vec{B}$ son las diadas del campo eléctrico y magnético respectivamente.

Sustituyendo las ecuaciones (3.6) y (3.7) en la ecuación (3.5), obtenemos la ecuación de balance de momento

$$
\nabla \cdot \mathbf{T}_{v}=\rho \vec{E}+\vec{J} \times \vec{B}+\frac{\partial}{\partial t} \epsilon_{0}(\vec{E} \times \vec{B}),
$$

donde

$$
\mathbf{T}_{v}=\left(\epsilon_{0} \vec{E} \vec{E}+\frac{1}{\mu_{0}} \vec{B} \vec{B}\right)-(1 / 2)\left(\epsilon_{0} \vec{E} \cdot \vec{E}+\frac{1}{\mu_{0}} \vec{B} \cdot \vec{B}\right) .
$$

Es de hacer notar que esta ecuación de balance de momento fue obtenida unicamente con las ecuaciones de Maxwell y el cálculo vectorial, en esta ecuación se observa la relación entre el tensor y las fuerzas del sistema, al utilizar este método alterno evitamos las inconsistencias que presenta el método usual. 


\section{Capítulo 4}

\section{Balance de momento electromagnético en medios materiales}

El problema de deducir la ecuación de balance de momento electromagnético en medios materiales es más complicado que en el vacío, de hecho, Jackson hace un comentario respecto a ese punto "El tema de los balances de energía y momento para la materia en campos electromagnéticos es evidentemente complicado. Remitamos al lector, para más cuestiones a Landau y Lifshitz [10] y también A Stratton [1]". El método estándar para deducir la ecuación de balance de momento electromagnético en medios materiales consiste básicamente en dos puntos, primero, dado que no se cuenta con una densidad de fuerza análoga a la de Lorentz se tiene que deducir una densidad de fuerza en el medio y una vez deducida esta densidad de fuerza el segundo punto es deducir la ecuación de balance de momento.

\subsection{Balance de momento electrostático en medios materiales fluidos. La densidad de fuerza de Helm- holtz}

En esta sección presentamos punto a punto la forma estándar de como se deduce la ecuación de balance de momento electrostático de un fluido, esta forma usual es seguida por muchos autores.

La forma estándar que siguen los autores para deducir la densidad de fuerza en medios es partir de la siguiente ecuación

$$
\frac{d U}{d t}=-\int \vec{f} \cdot \vec{v} d V
$$

la cual establece la relación entre energía libre del medio $U$, la densidad de fuerza del 
medio $\vec{f}$ y la velocidad de un elemento del medio $\vec{v}$.

Considerando el caso donde unicamente actúan campos electrostáticos, se define usualmente la energía libre electrostática del sistema, lo cual no es un punto trivial. Sin una discusión previa aceptan que la energía libre electrostática del sistema, es de la siguiente forma

$$
U_{E}=\frac{1}{2} \int \vec{E} \cdot \vec{D} d V,
$$

con esto escriben la variación de la energía del sistema con respecto al tiempo como

$$
\frac{d U_{E}}{d t}=-\frac{1}{2} \int \epsilon_{0} E^{2} \frac{\partial \epsilon_{r}}{\partial t} d V+\int \phi \frac{\partial \rho}{\partial t} d V,
$$

donde han hecho las siguientes consideraciones. Primero consideran que están en un caso electrostático, es decir

$$
\nabla \times \vec{E}=0,
$$

segundo que hay cargas libres

$$
\nabla \cdot \vec{D}=\rho
$$

y finalmente la ecuación constitutiva de la siguiente forma

$$
\vec{D}=\epsilon_{0} \epsilon_{r} \vec{E} .
$$

Con el fin de comparar las ecuaciones (4.1) y (4.3) introducen las ecuaciones de conservación de la carga y de la masa

$$
\nabla \cdot(\rho \vec{v})+\frac{\partial \rho}{\partial t}=0
$$

y

$$
\nabla \cdot\left(\rho_{m} \vec{v}\right)+\frac{\partial \rho_{m}}{\partial t}=0,
$$

además de las derivadas totales

$$
\frac{D \epsilon_{r}}{D t}=\frac{\partial \epsilon_{r}}{\partial t}+\nabla \epsilon_{r} \cdot \vec{v}
$$

y

$$
\frac{D \rho_{m}}{D t}=\frac{\partial \rho_{m}}{\partial t}+\nabla \rho_{m} \cdot \vec{v} .
$$

Aquí una vez más hacen una consideración importante, consideran que están en un medio material en el cual la permitividad sólo depende de la posición y la densidad del medio, es decir

$$
\epsilon_{r}=\epsilon_{r}\left(\vec{r}, \rho_{m}\right),
$$


tomando en cuenta esta consideración

$$
\frac{D \epsilon_{r}}{D t}=\frac{\partial \epsilon_{r}}{\partial \rho_{m}}\left(\frac{D \rho_{m}}{D t}\right)
$$

lo cual les permite escribir la siguiente ecuación

$$
\begin{aligned}
\frac{\partial \epsilon_{r}}{\partial t} & =\frac{D \epsilon_{r}}{D t}-\nabla \epsilon_{r} \cdot \vec{v} \\
& =-\frac{\partial \epsilon_{r}}{\partial \rho_{m}} \rho_{m}(\nabla \cdot \vec{v})-\nabla \epsilon_{r} \cdot \vec{v}
\end{aligned}
$$

sustituyendo las ecuaciones (4.7) y (4.13) en la ecuación (4.3) resulta

$$
\frac{d U_{E}}{d t}=\int\left[\rho \nabla \phi \cdot \vec{v}-\frac{\epsilon_{0}}{2} \nabla\left(E^{2} \rho_{m} \frac{\partial \epsilon_{r}}{\partial \rho_{m}}\right) \cdot \vec{v}+\frac{\epsilon_{0}}{2} E^{2} \nabla \epsilon_{r} \cdot \vec{v}\right] d V .
$$

Comparando las ecuaciones (4.1) y (4.14) reconocen una expresión para la densidad de fuerza $\vec{f}$, la cual es conocida como la densidad de fuerza de Helmholtz, que usualmente es denotada como $\vec{f}_{H}$ y tiene la siguiente forma

$$
\vec{f}_{H}=\rho \vec{E}+\frac{\epsilon_{0}}{2} \nabla\left(E^{2} \rho_{m} \frac{\partial \epsilon_{r}}{\partial \rho_{m}}\right)-\frac{\epsilon_{0}}{2} E^{2} \nabla \epsilon_{r}
$$

Precisamente $\vec{f}_{H}$ es la densidad de fuerza en un medio cuya permitividad solo depende de las cantidades señaladas en la igualdad (4.11), este resultado ha sido obtenido siguiendo este método por diversos autores como Stratton [1], Panofsky y Phillips [2], Tamm [9], Landau y Lifshitz [10] entre otros, ademas coincide con los resultados obtenidos mediante otros métodos, por ejemplo Gordon [5]. Una vez superado el primer punto, es decir, ya habiendo deducido una densidad de fuerza para el medio, se procede a realizar el balance considerando que la densidad de fuerza y el tensor de esfuerzos se relacionan de la siguiente forma

$$
f_{i}=\frac{\partial T_{i j}}{\partial r_{j}}
$$

en particular esta ecuación establece la relación entre las componentes de la densidad de fuerza del medio $f_{i}$ y las componentes del tensor de esfuerzos $T_{i j}$.

Una vez dicho esto y para facilitar el análisis de la expresión (4.15), separan la densidad de fuerza $\vec{f}_{H}$ de la siguiente manera

$$
\vec{f}_{H}=\vec{f}^{(1)}+\vec{f}^{(2)}
$$

donde

$$
\vec{f}^{(1)}=\rho \vec{E}-\frac{\epsilon_{0}}{2} E^{2} \nabla \epsilon_{r}
$$


$\mathrm{y}$

$$
\vec{f}^{(2)}=\frac{\epsilon_{0}}{2} \nabla\left(E^{2} \rho_{m} \frac{d \epsilon_{r}}{d \rho_{m}}\right) .
$$

$\vec{f}^{(1)}$ es la parte de la densidad de fuerza que encontró Maxwell, la cual depende de la variación de permitividad con respecto a la posición y de las cargas libres, así mismo $\vec{f}^{(2)}$ es la parte de la densidad de fuerza que depende del cambio de la permitividad con respecto a la densidad del medio.

A partir de este punto para facilitar el manejo de las ecuaciones usaremos la notación indicial.

Después de separar en dos partes a la densidad de fuerza $\vec{f}_{H}$ analizan por separado las partes de ésta. Considerando la ecuación (4.5) escriben la primera parte de esta densidad como

$$
f_{i}^{(1)}=E_{i} \frac{\partial D_{j}}{\partial r_{j}}-\frac{\epsilon_{0}}{2} E^{2} \frac{\partial \epsilon_{r}}{\partial r_{i}}
$$

Usando las siguientes igualdades

$$
\frac{\partial\left(E_{i} D_{j}\right)}{\partial r_{j}}-D_{j} \frac{\partial E_{i}}{\partial r_{j}}=E_{i} \frac{\partial D_{j}}{\partial r_{j}},
$$

y

$$
\frac{\partial\left(\epsilon_{r} E^{2}\right)}{\partial r_{i}}-\epsilon_{r} \frac{\partial E^{2}}{\partial r_{i}}=E^{2} \frac{\partial \epsilon_{r}}{\partial r_{i}},
$$

escriben la ecuación (4.20) como

$$
f_{i}^{(1)}=\frac{\partial}{\partial r_{j}}\left[E_{i} D_{j}-\frac{1}{2}(\vec{D} \cdot \vec{E}) \delta_{i j}\right]
$$

lo cual les permite escribir el tensor $T_{i j}^{(1)}$ asociado a la fuerza $\vec{f}^{(1)}$ como

$$
T_{i j}^{(1)}=E_{i} D_{j}-\frac{1}{2}(\vec{D} \cdot \vec{E}) \delta_{i j}
$$

el cual es precisamente el tensor encontrado por Maxwell.

Con lo que respecta a la parte de la densidad de fuerza $\overrightarrow{f^{(2)}}$, escriben el tensor asociado $T_{i j}^{(2)}$ directamente como

$$
T_{i j}^{(2)}=\frac{\epsilon_{0}}{2} E^{2} \rho_{m} \frac{\partial \epsilon_{r}}{\partial \rho_{m}} \delta_{i j},
$$

al sumar los tensores $T_{i j}^{(1)}$ y $T_{i j}^{(2)}$ obtienen

$$
\begin{aligned}
T_{i j} & =T_{i j}^{(1)}+T_{i j}^{(2)} \\
& =\left[E_{i} D_{j}-\frac{1}{2}(\vec{D} \cdot \vec{E}) \delta_{i j}\right]+\frac{\epsilon_{0}}{2} E^{2} \rho_{m} \frac{\partial \epsilon_{r}}{\partial \rho_{m}} \delta_{i j},
\end{aligned}
$$


donde el tensor $T_{i j}$, es el tensor asociado a la densidad de fuerza de Helmholtz $\vec{f}_{H}$, el cual está compuesto por la suma de dos tensores como señala Tamm [9].

Una vez encontrado $T_{i j}$ y considerando la ecuación (4.16) se puede escribir el balance de momento como

$$
\left(\vec{f}_{H}\right)_{i}=\frac{\partial T_{i j}}{\partial r_{j}}
$$

es decir

$$
\rho E_{i}-\frac{\epsilon_{0}}{2} E^{2} \frac{\partial \epsilon_{r}}{\partial r_{i}}+\frac{\epsilon_{0}}{2} \frac{\partial}{\partial r_{i}}\left(E^{2} \rho_{m} \frac{\partial \epsilon_{r}}{\partial \rho_{m}}\right)=\frac{\partial}{\partial r_{j}}\left[E_{i} D_{j}-\frac{1}{2}(\vec{D} \cdot \vec{E}) \delta_{i j}+\frac{\epsilon_{0}}{2} E^{2} \rho_{m} \frac{\partial \epsilon_{r}}{\partial \rho_{m}} \delta_{i j}\right] .
$$

El balance discutido se sustenta en dos hipótesis, primero, que la variación de energía nos conduce a una fuerza y segundo que la energía libre electrostática del sistema es de la forma escrita en la ecuación (4.2). Una vez que los obtienen la densidad de fuerza no escriben explícitamente una ecuación de balance de momento, tal parece que no están interesados en la estructura general de la ecuación de balance de momento.

Por otra parte podemos cuestionarnos que tan general es esta densidad de fuerza obtenida y por consiguiente el balance que se construye con ella y ¿Cómo es la ecuación de balance de momento para un medio con otras características?

\subsection{Balance de momento electromagnético en me- dios materiales sólidos}

Hasta ahora hemos analizado la densidad de fuerza para el caso en el que la permitividad depende únicamente de la posición y de la densidad del medio, es decir el caso de un fluido, pero no hemos visto la forma que tiene dicha densidad de fuerza cuando el medio material es un sólido. Puesto que las propiedades dieléctricas de un sólido varían no sólo al cambiar su densidad (como en el caso de un fluido) sino también con las deformaciones que no modifican el volumen, es necesario hacer un análisis de la permitividad para considerar la nuevas cantidades de las que depende. El método usual para resolver este problema sigue una estructura similar al usado para deducir la densidad de fuerza cuando el medio es un fluido, autores como Stratton, Robinson, Landau y Lifshitz hacen este tipo de deducciones para obtener una densidad de fuerza para un sólido.

Para mostrar la forma que tiene esta densidad de fuerza seguimos el método usado por Robinson, por su notación más moderna y concisa que facilita la comprensión de los argumentos, más adelante debido a la importancia del problema haremos una comparación entre los resultados obtenidos por Robinson [4] y Stratton [1]. 
Robinson, para deducir la densidad de fuerza parte de la expresión para el cambio de energía libre electrostática del sistema

$$
\frac{d U_{E}}{d t}=\int\left[-\rho E_{i} v_{i}-\frac{\epsilon_{0}}{2} E_{i} E_{j} \frac{\partial \epsilon_{i j}}{\partial t}\right] d V
$$

la cual es análoga a (4.3). En la ecuación (4.29) se ha supuesto que la relación constitutiva es

$$
D_{i}=\epsilon_{0} \epsilon_{i j} E_{j}
$$

además, análogamente al caso de un fluido considera que hay cargas libres y que se conserva la carga, es decir que se cumplen las ecuaciones (4.5) y (4.7). Una vez hechas estas consideraciones se enfoca en analizar el término $\frac{\partial \epsilon_{i j}}{\partial t}$. Considera que la permitividad $\epsilon_{i j}$, es un tensor de segundo rango que depende de las tensiones $S_{i j} \mathrm{y}$ rotaciones $W_{i j}$ del sistema

$$
\epsilon_{i j} \equiv \epsilon_{i j}\left(S_{i j}, W_{i j}\right)
$$

Las cantidades $S_{i j}$ y $W_{i j}$ son la parte simétrica y antisimetrica del tensor

$$
\begin{aligned}
\frac{\partial u_{i}}{\partial r_{j}} & =\frac{1}{2}\left(\frac{\partial u_{i}}{\partial r_{j}}+\frac{\partial u_{j}}{\partial r_{i}}\right)-\frac{1}{2}\left(\frac{\partial u_{j}}{\partial r_{i}}-\frac{\partial u_{i}}{\partial r_{j}}\right) \\
& =S_{i j}-W_{i j}
\end{aligned}
$$

donde $u_{i}$ representa la posición de los componentes del medio.

Con estas consideraciones y después de un proceso muy laborioso y poco claro se escribe

$$
\frac{\partial \epsilon_{i j}}{\partial t}=\frac{1}{2} \epsilon_{i k}\left(\frac{\partial v_{j}}{\partial r_{k}}-\frac{\partial v_{k}}{\partial r_{j}}\right)+\frac{1}{2} \epsilon_{k j}\left(\frac{\partial v_{i}}{\partial r_{k}}-\frac{\partial v_{k}}{\partial r_{i}}\right)+\alpha_{i j k l} \frac{\partial v_{k}}{\partial r_{l}}-v_{k} \frac{\partial \epsilon_{i j}}{\partial r_{k}}
$$

donde

$$
\alpha_{i j k l} \equiv \frac{\partial \epsilon_{i j}}{\partial S_{k l}}
$$

Ahora se sustituye la ecuación (4.33) en la expresión (4.29) y después de una laboriosa manipulación se obtiene la siguiente expresión

$$
\begin{gathered}
\frac{d U}{d t}=\int\left[-\rho E_{i} v_{i}+\frac{1}{4} v_{j} \frac{\partial\left(E_{j} D_{k}\right)}{\partial r_{k}}-\frac{1}{4} v_{k} \frac{\partial\left(E_{j} D_{k}\right)}{\partial r_{j}}+\frac{1}{4} v_{i} \frac{\partial\left(E_{i} D_{k}\right)}{\partial r_{k}}-\right. \\
\left.\frac{1}{4} v_{k} \frac{\partial\left(E_{i} D_{k}\right)}{\partial r_{i}}+v_{k} \frac{\partial\left(\frac{\epsilon_{0}}{2} E_{i} E_{j} \alpha_{i j k l}\right)}{\partial r_{l}}+v_{k} \frac{\epsilon_{0}}{2} E_{i} E_{j} \frac{\partial \epsilon_{i j}}{\partial r_{k}}\right] d V
\end{gathered}
$$

renombrando índices y factorizando $v_{i}$ se reconoce a la densidad de fuerza

$$
f_{i(s)}=\rho E_{i}-\frac{1}{2} \frac{\partial}{\partial r_{j}}\left(E_{i} D_{j}-E_{j} D_{i}\right)+\frac{\partial T_{i j}^{e s}}{\partial r_{j}}+\frac{\epsilon_{0}}{2} E_{k} E_{l} \frac{\partial \epsilon_{k l}}{\partial r_{i}}
$$


donde

$$
T_{i j}^{e s} \equiv-\frac{\epsilon_{0}}{2} E_{k} E_{l} \alpha_{k l i j} .
$$

$f_{i(s)}$ es la densidad de fuerza sobre un sólido debida a campos electrostáticos, la cual se diferencia con la densidad de fuerza en un fluido $f_{i(H)}$ por el término $\frac{\partial T_{i j}^{e s}}{\partial r_{j}}$. Precisamente $T_{i j}^{e s}$ es el tensor propuesto por Robinson [4], y que como veremos más adelante coincide con los resultados encontrados previamente por Stratton [1] para describir a los sólidos.

Con el propósito de escribir la densidad de fuerza $f_{i(s)}$ en términos de la divergencias de tensores, se analizan los términos de esta densidad de fuerza $f_{i(s)}$, se escriben los términos de $f_{i(s)}$ como

$$
\rho E_{i}=\frac{\partial D_{j}}{\partial r_{j}} E_{i}
$$

y

$$
\frac{\epsilon_{0}}{2} E_{k} E_{l} \frac{\partial \epsilon_{k l}}{\partial r_{i}}=-\frac{1}{2}\left(E_{j} \frac{\partial D_{j}}{\partial r_{i}}-D_{j} \frac{\partial E_{j}}{\partial r_{i}}\right) .
$$

$\mathrm{Al}$ sustituir las igualdades (4.38) y (4.39) en la ecuación (4.36) se obtiene que

$$
f_{i(s)}=\frac{\partial}{\partial r_{j}}\left(E_{i} D_{j}-\frac{1}{2} E_{i} D_{j}+\frac{1}{2} E_{j} D_{i}+T_{i j}^{e s}\right)-\frac{1}{2}\left(E_{j} \frac{\partial D_{j}}{\partial r_{i}}-D_{j} \frac{\partial E_{j}}{\partial r_{i}}\right),
$$

finalmente

$$
f_{i(s)}=\frac{\partial}{\partial r_{j}}\left[\frac{1}{2}\left(E_{i} D_{j}+E_{j} D_{i}-\vec{E} \cdot \vec{D} \delta_{i j}\right)+T_{i j}^{e s}\right] .
$$

Para continuar se define

$$
T_{i j}^{e} \equiv \frac{1}{2}\left[E_{i} D_{j}+E_{j} D_{i}-(\vec{E} \cdot \vec{D}) \delta_{i j}\right]
$$

entonces

$$
f_{i(s)}=\frac{\partial}{\partial r_{j}}\left(T_{i j}^{e}+T_{i j}^{e s}\right),
$$

con lo cual se puede escribir finalmente la ecuación de balance de momento para un sólido como

$$
\rho E_{i}-\frac{1}{2} \frac{\partial}{\partial r_{j}}\left(E_{i} D_{j}-E_{j} D_{i}\right)+\frac{\partial T_{i j}^{e s}}{\partial r_{j}}+\frac{\epsilon_{0}}{2} E_{k} E_{l} \frac{\partial \epsilon_{k l}}{\partial r_{i}}=\frac{\partial}{\partial r_{j}}\left(T_{i j}^{e}+T_{i j}^{e s}\right) .
$$

La ecuación (4.44) establece la relación entre la densidad de fuerzas en un sólido y su tensor de esfuerzos, es importante mencionar que esta expresión no la escribe Robinson de manera explícita, tal como sucede en el caso de los fluidos, parece no 
estar interesado en la estructura general del balance. Por otra parte podemos darnos cuenta que las limitaciones e hipótesis de este método son análogas a las del método usado en el caso de un fluido, debido a la estructura similar de los procedimientos. Con esto queremos decir que en esencia estos dos métodos son iguales salvo cuando consideran las cantidades de las que depende la permitividad del medio, ambos métodos se sustenta en dos hipótesis, primero, que la variación de energía libre conduce a una fuerza y segundo que la energía libre del sistema es de la forma de (4.2).

Si aceptamos que estos dos métodos son en esencia iguales podemos concluir que debería haber una relación entre la densidad de fuerza en un fluido $f_{i(H)}$ y la densidad de fuerza en un sólido $f_{i(s)}$ puesto que las características de un fluido son un caso particular de las características de un sólido. Por otra parte al igual que en el caso de un fluido podemos preguntarnos si existe un método alterno para obtener la densidad de fuerza $f_{i(s)}$ el cual supere las limitaciones del método estándar y si es que existe este método alterno ¿Que forma tiene la ecuación de balance de momento para un sólido?

\subsection{Densidad de fuerza en campos magnetostáticos. Método de Stratton}

En las secciones anteriores analizamos la forma usual de deducir las densidades de fuerza sobre sólidos y fluidos debidas a un campo electrostático. El propósito de esta sección es mostrar la deducción usual de la densidad de fuerza sobre un sólido en presencia únicamente de un campo magnetostático.

Para mostrar la forma usual de deducir esta densidad de fuerza, seguiremos el desarrollo realizado por Stratton [1] pero con un lenguaje distinto, además de añadir algunos desarrollos, con el fin de mostrar la estructura de esta deducción y facilitar la comparación con otros autores.

La ecuación constitutiva entre $\vec{B}$ y $\vec{H}$ para un sólido, es

$$
B_{j}=\mu_{0} \mu_{j k} H_{k}
$$

con esto la energía libre magnética $U_{m}$, se expresa como

$$
U_{m}=\frac{1}{2} \int \mu_{0} \mu_{j k} H_{j} H_{k} d V
$$

y su variación como

$$
\delta U_{m}=\frac{1}{2} \int \mu_{0}\left(\delta \mu_{j k}\right) H_{j} H_{k} d V .
$$

Al considerar que la permeabilidad $\mu_{j k}$ depende, de la posición $\vec{r}$ y el tensor de tensiones simétrico $S_{l m}$, es decir

$$
\mu_{j k} \equiv \mu_{j k}\left(r_{i}, S_{l m}\right)
$$


la variación de la permeabilidad es

$$
\delta \mu_{j k}=\delta r_{k} \frac{\partial \mu_{j k}}{\partial r_{k}}+\delta S_{l m} \frac{\partial \mu_{j k}}{\partial S_{l m}}
$$

Para continuar, se define el tensor

$$
b_{j k l m} \equiv \frac{\partial \mu_{j k}}{\partial S_{l m}}
$$

con ayuda de las ecuaciones (4.49) y (4.50) se escribe la ecuación (4.47) como

$$
\begin{aligned}
\delta U_{m} & =\frac{1}{2} \int \mu_{0} H_{j} H_{k}\left[\delta r_{i} \frac{\partial \mu_{j k}}{\partial r_{i}}+b_{j k l m} \delta S_{l m}\right] d V \\
& =\frac{1}{2} \int \mu_{0} H_{j} H_{k} \delta r_{i} \frac{\partial \mu}{\partial r_{i}} \delta_{j k} d V+\frac{1}{2} \int \mu_{0} H_{j} H_{k} b_{j k l m} \delta S_{l m} d V \\
& =\delta U_{m t}+\delta U_{m s},
\end{aligned}
$$

donde $\delta U_{m t}$ es la variación de la energía asociada con una traslación infinitesimal $\delta r_{i}$, la otra parte $\delta U_{m s}$ es la parte que representa una tensión pura asociada con la deformación, es claro entonces que

$$
\delta U_{m s}=\frac{1}{2} \int \mu_{0} H_{j} H_{k} b_{j k l m} \delta S_{l m} d V
$$

$\mathrm{y}$

$$
\delta U_{m t}=\frac{1}{2} \int \mu_{0} H_{k} H_{k} \delta r_{i} \frac{\partial \mu}{\partial r_{i}} d V .
$$

Si $U_{m(e l a s)}$ es la energía elástica, y considerando que dicha energía depende de la tensión, es decir

$$
U_{m(\text { elas })}=U_{m(\text { elas })}\left(S_{l m}\right)
$$

la variación de la energía elástica $U_{m(\text { elas })}$ tiene la siguiente forma

$$
\delta U_{m(\text { elas })}=\frac{1}{2} \int \frac{\partial u_{m(\text { elas })}}{\partial S_{l m}} \delta S_{l m} d V
$$

tomando en cuenta que la energía libre magnética $U_{m}$ del sistema esta compuesta de tres partes, es decir, la energía elástica $U_{m(e l a s)}$, la energía asociada con la deformación del material $U_{m s}$ y la energía asociada con una traslación infinitesimal $U_{m t}$, se puede escribir las variación de la energía libre magnética como

$$
\delta U_{m}=\delta\left(U_{m(\text { elas })}+U_{m s}+U_{m t}\right) .
$$

Dado que $U_{m}$ es la energía libre magnética libre del sistema, el trabajo requerido para llevar acabo un pequeño desplazamiento o deformación de un cuerpo en un campo magnético se escribe como

$$
\delta W=-\delta U_{m}
$$


Por otra parte considerando que bajo la influencia de fuerzas externas los elementos de un fluido o de un sólido elástico pueden efectuar desplazamientos relativos. El trabajo realizado por las fuerzas para desplazar cada punto del medio una distacia $\delta \vec{s}$ es

$$
\delta W=\int \vec{f} \cdot \delta \vec{s} d V+\int \vec{t} \cdot \delta \vec{s} d a
$$

donde las los vectores $\vec{f} \mathrm{y} \vec{t}$, usualmente son denominados como densidades de fuerzas volumétricas y densidades de fuerzas superficiales respectivamente. Con las ecuaciones (4.57) y (4.58) se escribe la variación de la energía libre magnética como

$$
\int \vec{f} \cdot \delta \vec{s} d V+\int \vec{t} \cdot \delta \vec{s} d a=-\delta U_{m}
$$

sustituyendo la ecuación (4.56) en la ecuación (4.59), se obtiene que

$$
\int \vec{f} \cdot \delta \vec{s} d V+\int \vec{t} \cdot \delta \vec{s} d a=-\delta\left(U_{m(\text { elas })}+U_{m s}+U_{m t}\right)
$$

Es conveniente considerar que las densidades de fuerza $\vec{f}$ y $\vec{t}$ están compuestos de dos partes, la parte 1 se refiere a la fuerza en el material antes de la deformación y la parte 2 se refiere a después de la deformación, entonces

$$
\vec{f}=\overrightarrow{f_{1}}+\overrightarrow{f_{2}}
$$

y

$$
\vec{t}=\overrightarrow{t_{1}}+\overrightarrow{t_{2}}
$$

Considerando que el estado inicial el cuerpo está sin deformar, se puede escribir

$$
\int \overrightarrow{f_{1}} \cdot \delta \vec{s} d V+\int \overrightarrow{t_{1}} \cdot \delta \vec{s} d a=-\delta U_{m t}
$$

al considerar que antes de la deformación $\overrightarrow{t_{1}}=0$ y $\delta \vec{r}=\delta \vec{s}$, se tiene que

$$
\int \vec{f}_{1} \cdot \delta \vec{s} d V=-\delta U_{m t}=\int\left[-\frac{1}{2} \mu_{0} H^{2} \nabla \mu\right] \cdot \delta \vec{s} d V
$$

entonces se encuentra que la densidad de fuerza $\vec{f}_{1}$ es

$$
\overrightarrow{f_{1}}=-\frac{1}{2} \mu_{0} H^{2} \nabla \mu
$$

Para el cuerpo después de la deformación se tiene que

$$
\int \overrightarrow{f_{2}} \cdot \delta \vec{s} d V+\int \overrightarrow{t_{2}} \cdot \delta \vec{s} d a=-\delta\left(U_{m(\text { elas })}+U_{m s}\right),
$$

es decir

$$
\int \overrightarrow{f_{2}} \cdot \delta \vec{s} d V+\int \overrightarrow{t_{2}} \cdot \delta \vec{s} d a=-\int\left(\frac{1}{2} \mu_{0} b_{j k l m} H_{j} H_{k} \delta S_{l m}+\frac{\partial u_{m(e l a s)}}{\partial S_{l m}} \delta S_{l m}\right) d V .
$$


Es conveniente mencionar que Stratton [1] no escribe la ecuación (4.67) de esta forma, esto se debe además de usar una notación distinta, a dos razones, primero a que hace una analogía del tensor $b_{j k l m}$ con el tensor $\alpha_{j k l m}$, entonces considera que el tensor $b_{j k l m}$ contiene únicamente dos constantes independientes, así pues escribe el término $b_{j k l m} H_{j} H_{k}$ en un caso particular y segundo considera una forma particular de $u_{m(e l a s)}$, como

$$
u_{m(\text { elas })}=\frac{1}{2} \lambda_{1} S_{i i} S_{i i}+\lambda_{2} S_{i m} S_{i m}
$$

$\mathrm{Al}$ considerar esta forma particular de $u_{m \text { (elas) }}$, escribe una expresión particular para el término $\frac{\partial u_{m(e l a s)}}{\partial S_{l m}}$. La importancia que tiene escribir el balance de la forma dada en la ecuación (4.67) se verá al final de esta deducción.

Tomando en cuenta la ecuación (4.58), se escribe la variación del trabajo de la siguiente manera

$$
\delta W_{2}=\int \overrightarrow{f_{2}} \cdot \delta \vec{s} d V+\int \overrightarrow{t_{2}} \cdot \delta \vec{s} d a
$$

por otra parte, la densidad de energía libre magnética $u_{m}$ depende, en cualquier punto, del estado local de tensión, por lo tanto, se supone que

$$
u_{m}=u_{m}\left(S_{l m}\right)
$$

entonces

$$
\delta U_{m}\left(S_{l m}\right)=\int\left(\frac{\partial u_{m}}{\partial S_{l m}}\right) \delta S_{l m} d V=\int T_{l m} \delta S_{l m} d V
$$

además, de acuerdo con la ecuación (4.57), se puede escribir

$$
\delta W_{2}=\delta U_{m}\left(S_{l m}\right)
$$

Con ayuda de la ecuaciones (4.71) y (4.72), se escribe la ecuación (4.69) como

$$
\int \overrightarrow{f_{2}} \cdot \delta \vec{s} d V+\int \overrightarrow{t_{2}} \cdot \delta \vec{s} d a=\int T_{l m} \delta S_{l m} d V
$$

sustituyendo la ecuación (4.67) en la ecuación (4.73) resulta

$$
-\int\left(\frac{1}{2} \mu_{0} b_{j k l m} H_{j} H_{k} \delta S_{l m}+\frac{\partial u_{m(\text { elas })}}{\partial S_{l m}} \delta S_{l m}\right) d V=\int T_{l m} \delta S_{l m} d V
$$

con lo cual se obtiene que

$$
-\left(\frac{1}{2} \mu_{0} b_{j k l m} H_{j} H_{k}+\frac{\partial u_{m(\text { elas })}}{\partial S_{l m}}\right)=T_{l m} .
$$

Ahora en esta deducción se considera que la componente $l$ de la densidad de fuerza se escribe como

$$
f_{l}=\frac{\partial T_{l m}}{\partial r_{m}}
$$


Usando la ecuación (4.76) en la ecuación (4.75) se obtiene

$$
f_{2 l}=\frac{\partial}{\partial r_{m}}\left(-\frac{1}{2} \mu_{0} b_{j k l m} H_{j} H_{k}-\frac{\partial u_{m(\text { elas })}}{\partial S_{l m}}\right) .
$$

Considerando la analogía con el caso electrostático donde se consideró que únicamente existían fuerzas de origen electrostático, se considera que

$$
\frac{\partial}{\partial r_{m}}\left(-\frac{\partial u_{m(\text { elas })}}{\partial S_{l m}}\right)=0
$$

entonces se obtiene para la densidad de fuerza

$$
f_{2 l}=\frac{\partial}{\partial r_{m}}\left(-\frac{1}{2} \mu_{0} b_{j k l m} H_{j} H_{k}\right)
$$

Sumando las densidades de fuerza $\vec{f}_{1}$ y $f_{2 l}$, se obtiene la densidad de fuerza total del sistema

$$
f_{l}=-\frac{1}{2} \mu_{0} H^{2} \frac{\partial \mu}{\partial r_{l}}+\frac{\partial}{\partial r_{m}}\left(-\frac{1}{2} \mu_{0} b_{j k l m} H_{j} H_{k}\right) .
$$

Aquí es importante hacer notar que el haber usado esta de notación, diferente de la de Stratton, nos permite escribir a la densidad de fuerza $f_{l}$ en la ecuación (4.80). Esto es provechoso pues así facilita considerablemente al lector moderno su comprensión y nos permite comparar claramente con resultados obtenidos por otros autores como Robinson [4] y Landau y Lifshitz [10].

Así en completa analogía con el caso electrostático, se deduce que la densidad de fuerza ejercida sobre un sólido debida a un campo magnetostático es

$$
f_{l}=(\vec{j} \times \vec{B})_{l}-\frac{1}{2} \mu_{0} H^{2} \frac{\partial \mu}{\partial r_{l}}-\frac{\partial}{\partial r_{m}}\left(\frac{1}{2} \mu_{0} b_{j k l m} H_{j} H_{k}\right)
$$

donde se ha considerado que en el medio puede circular una densidad de corriente $\vec{j}$ y que la permeabilidad es independiente de $\vec{H}$.

Es claro ver la estructura similar que presentan las ecuaciones (4.36) y (4.81), cabe señalar que los tensores $b_{j k l m}$ y $\alpha_{k l i j}$ se suponen con las mismas propiedades. las cuales fueron discutidas en la sección anterior.

El método seguido por Stratton para deducir la densidad de fuerza sobre un sólido en un caso magnetostático, es en esencia, de estructura similar a los seguidos en las secciones anteriores, en esta sección solo hemos analizado la densidad de fuerza debida a campos magnetostáticos en un sólido, con lo cual podría pensarse que olvidamos considerar cuando el medio es un fluido, más adelante veremos la relación que guardan estos medios. 
Deducción de la densidad de fuerza de Helmholtz como un paso particular de la densidad de fuerza en un sólido

\subsection{Deducción de la densidad de fuerza de Helm- holtz como un paso particular de la densidad de fuerza en un sólido}

Mostrar la relación que tienen la densidad de fuerza de Helmholtz y la densidad de fuerza en un sólido, es un tema en el cual se pone énfasis usualmente, esto puede ser una señal de que se busca una densidad de fuerza más general, puesto que $\vec{f}_{H}$ es un caso particular de $f_{i(s)}$.

Para mostrar esta relación analizamos la parte elástica del tensor elástico

$$
f_{i}^{e s} \equiv \frac{\partial}{\partial r_{j}} T_{i j}^{e s}=\frac{\partial}{\partial r_{j}}\left(-\frac{\epsilon_{0}}{2} E_{k} E_{l} \alpha_{k l i j}\right) .
$$

Landau y Lifshitz [10] hacen un análisis sobre el tensor $\alpha_{k l i j}$ donde mencionan que en el caso general es un tensor constante de cuarto orden, simétrico respecto de los pares de índices $k$ y $l, i$ y $j$. Así mismo Startton [1] hace una discusión detallada para mostrar que en el caso de un sólido el tensor $\alpha_{k l i j}$ sólo tiene dos coeficientes constantes independientes. El desarrollo de estas constantes se analiza en el apéndice. Tomando en cuenta esto podemos escribir una expresión para la permitividad de la siguiente forma

$$
\epsilon_{i j}=a_{1} S_{i j}+a_{2} S_{k k} \delta_{i j}
$$

donde $a_{1}$ y $a_{2}$ son constantes escalares independientes.

Una vez definida la expresión para la permitividad, podemos encontrar la forma particular de $\alpha_{i j k l}$ mediante la siguiente ecuación

$$
\begin{aligned}
\alpha_{i j k l} & =\frac{\partial \epsilon_{i j}}{\partial S_{k l}} \\
& =\frac{\partial}{\partial S_{k l}}\left(a_{1} S_{i j}+a_{2} S_{k k} \delta_{i j}\right),
\end{aligned}
$$

entonces, para un sólido

$$
\alpha_{k l i j}=a_{1} \delta_{k i} \delta_{j l}+a_{2} \delta_{i j} \delta_{k l} .
$$

Escribiendo a la densidad de fuerza $f_{i}^{e s}$ en términos de (4.85) tenemos que

$$
f_{i}^{e s}=\frac{\partial}{\partial r_{j}}\left[-\frac{\epsilon_{0}}{2} E_{k} E_{l}\left(a_{1} \delta_{k i} \delta_{j l}+a_{2} \delta_{i j} \delta_{k l}\right)\right]
$$

En el caso de un fluido, la constantes $a_{1}$ y $a_{2}$ son

$$
a_{1}=0
$$

$\mathrm{y}$

$$
a_{2}=\rho_{m} \frac{\partial \epsilon_{r}}{\partial \rho_{m}}
$$




\section{4.}

sustituyendo los valores de $a_{1}$ y $a_{2}$ en la densidad de fuerza (4.86)

$$
\begin{aligned}
f_{i}^{e s} & =-\frac{\epsilon_{0}}{2} \frac{\partial}{\partial r_{j}}\left[E_{k} E_{l}\left(\rho_{m} \frac{\partial \epsilon_{r}}{\partial \rho_{m}}\right) \delta_{i j} \delta_{k l}\right] \\
& =-\frac{\epsilon_{0}}{2} \frac{\partial}{\partial r_{j}}\left[E_{k} E_{k}\left(\rho_{m} \frac{\partial \epsilon_{r}}{\partial \rho_{m}}\right) \delta_{i j}\right] \\
& =-\frac{\epsilon_{0}}{2} \frac{\partial}{\partial r_{i}}\left(E^{2} \rho_{m} \frac{\partial \epsilon_{r}}{\partial \rho_{m}}\right) .
\end{aligned}
$$

Si escribimos la densidad de fuerza $f_{i}$ para este caso

$$
\begin{aligned}
f_{i} & =\rho E_{i}-\frac{1}{2} \frac{\partial}{\partial r_{j}} \epsilon_{0}\left[E_{i}\left(\epsilon_{r} E_{j}\right)-E_{j}\left(\epsilon_{r} E_{i}\right)\right]+\frac{\partial T_{i j}^{e s}}{\partial r_{j}}+\frac{\epsilon_{0}}{2} E_{k} E_{l} \frac{\partial \epsilon_{r}}{\partial r_{i}} \delta_{k l} \\
& =\rho E_{i}-\frac{\epsilon_{0}}{2} \frac{\partial}{\partial r_{i}}\left(E^{2} \rho_{m} \frac{\partial \epsilon_{r}}{\partial \rho_{m}}\right)+\frac{\epsilon_{0}}{2} E_{k} E_{k} \frac{\partial \epsilon_{r}}{\partial r_{i}} .
\end{aligned}
$$

Al comparar la ecuación anterior con la expresión (4.15), es fácil, reconocer que la expresión (4.90) es precisamente la densidad de fuerza de Helmholtz $\vec{f}_{H}$, lo cual es un resultado importante, puesto que muestra que la fuerza $f_{i(s)}$ para un sólido contiene, como caso particular la densidad de fuerza de un fluido $\vec{f}_{H}$.

Por otra parte en el caso de un fluido el tensor $T_{i j}^{s}$ se puede escribir simplemente como

$$
T_{i j}^{s}=\epsilon_{0} \epsilon_{r}\left(E_{i} E_{j}-\frac{1}{2} E^{2} \delta_{i j}\right),
$$

con lo cual podemos escribir el balance, en este caso particular, como

$$
\rho E_{i}-\frac{\epsilon_{0}}{2} \frac{\partial}{\partial r_{i}}\left(E^{2} \rho_{m} \frac{\partial \epsilon_{r}}{\partial \rho_{m}}\right)+\frac{\epsilon_{0}}{2} E^{2} \frac{\partial \epsilon_{r}}{\partial r_{i}}=\frac{\partial}{\partial r_{j}}\left[E_{i} D_{j}+\frac{1}{2} \epsilon_{0} E^{2}\left(\rho_{m} \frac{\partial \epsilon_{r}}{\partial \rho_{m}}-\epsilon_{r}\right) \delta_{i j}\right]
$$

Si pensamos en términos de generalidad, es claro que la densidad de fuerza de un sólido es más general que la densidad de fuerza de Helmholtz y por ende el balance construido con $f_{i(s)}$ también es más general que el construido con $\vec{f}_{H}$, pero ya hemos mostrado que nuestro balance propuesto contiene ambos densidades de fuerza como casos particulares siendo esto prueba de que indudablemente es un balance más general que los anteriores. 


\section{Capítulo 5}

\section{Balance de momento electromagnético en medios materiales. Deducción alternativa}

Partir de la energía libre del sistema para obtener la densidad de fuerza en el medio y posteriormente su tensor de esfuerzos, es un procedimiento que tiene sus complicaciones, por ello resulta factible analizar el balance de momento electromagnético desde otro enfoque.

En la búsqueda de un método alterno el cual evite pasar por la hipótesis que contiene el método usual Campos et al [16] propusieron una forma alterna de obtener la ecuación de balance de momento electromagnético en medios materiales, la cual tiene como fundamento las ecuaciones de Maxwell e identidades vectoriales y tensoriales, este método alterno presenta una forma clara y directa de obtener el balance.

Para obtener este balance, partimos de las ecuaciones de Maxwell en medios materiales

$$
\begin{gathered}
\nabla \cdot \vec{D}=\rho \\
\nabla \cdot \vec{B}=0 \\
\nabla \times \vec{E}=-\frac{\partial \vec{B}}{\partial t} \\
\nabla \times \vec{H}=\vec{j}+\frac{\partial \vec{D}}{\partial t} .
\end{gathered}
$$

Tomando como base estas ecuaciones y mediante multiplicaciones escalares y vectoriales podemos escribir las siguientes igualdades

$$
\begin{gathered}
\vec{E}(\nabla \cdot \vec{D})=\rho \vec{E}, \\
\vec{H}(\nabla \cdot \vec{B})=0,
\end{gathered}
$$




$$
-\vec{D} \times(\nabla \times \vec{E})=\vec{D} \times \frac{\partial \vec{B}}{\partial t}
$$

y

$$
-\vec{B} \times(\nabla \times \vec{H})=-\vec{B} \times\left(\vec{j}+\frac{\partial \vec{D}}{\partial t}\right) .
$$

Sumando las ecuaciones (5.5), (5.6), (5.7) y (5.8) obtenemos

$$
\vec{E}(\nabla \cdot \vec{D})+\vec{H}(\nabla \cdot \vec{B})-\vec{D} \times(\nabla \times \vec{E})-\vec{B} \times(\nabla \times \vec{H})=\rho \vec{E}+\vec{j} \times \vec{B}+\frac{\partial}{\partial t}(\vec{D} \times \vec{B}) .
$$

La ecuación (5.9), claramente se obtiene a partir de las ecuaciones de Maxwell y tiene como característica importante, el hecho de que para deducirla no se hizo uso de ninguna hipótesis, en otras palabras es una ecuación deducida con consideraciones puramente de la teoría electromagnética.

Ahora, con el fin de analizar la ecuación (5.9), utilizamos identidades vectoriales y tensoriales para reescribir los miembros de la parte izquierda de esta ecuación, de tal forma que

$$
\vec{E}(\nabla \cdot \vec{D})-\vec{D} \times(\nabla \times \vec{E})=\nabla \cdot\left[\vec{D} \vec{E}-\frac{1}{2}(\vec{D} \cdot \vec{E}) \mathbf{I}\right]+\frac{1}{2}[(\nabla \vec{D}) \cdot \vec{E}-(\nabla \vec{E}) \cdot \vec{D}]
$$

y

$$
\vec{H}(\nabla \cdot \vec{B})-\vec{B} \times(\nabla \times \vec{H})=\nabla \cdot\left[\vec{B} \vec{H}-\frac{1}{2}(\vec{B} \cdot \vec{H}) \mathbf{I}\right]+\frac{1}{2}[(\nabla \vec{B}) \cdot \vec{H}-(\nabla \vec{H}) \cdot \vec{B}] .
$$

Sustituyendo las identidades (5.10) y (5.11) en la ecuación (5.9) y definiendo

$$
\mathbf{T}_{E M}=(\vec{D} \vec{E}+\vec{B} \vec{H})-\frac{1}{2}(\vec{D} \cdot \vec{E}+\vec{B} \cdot \vec{H}) \mathbf{I}
$$

obtenemos

$$
\begin{aligned}
& \nabla \cdot \mathbf{T}_{E M}=\rho \vec{E}+\vec{j} \times \vec{B}+\frac{\partial}{\partial t}(\vec{D} \times \vec{B}) \\
& \quad+\frac{1}{2}[(\nabla \vec{E}) \cdot \vec{D}-(\nabla \vec{D}) \cdot \vec{E}+(\nabla \vec{H}) \cdot \vec{B}-(\nabla \vec{B}) \cdot \vec{H}]
\end{aligned}
$$

Con esto presentamos un método alterno para obtener la ecuación de balance en medios, la solidez de este método consiste en que está sustentando únicamente por dos pilares, las ecuaciones de Maxwell y el cálculo vectorial y tensorial.

La ecuación (5.13) es, precisamente, una ecuación general de balance de momento electromagnético en medios materiales, la cual por si sola ya representa un resultado importante y merece una discusión a fondo, puesto que no existen resultados previos donde se haya obtenido una ecuación de balance general. Como ya hemos 
visto en las secciones anteriores, la manera estándar de deducir la ecuación de balance es presentada como un caso exclusivo de cada medio y nunca como un caso general.

Una forma de discutir la generalidad de la ecuación (5.13), es analizar el término del miembro derecho de esta ecuación, el cual es una densidad de fuerza. Este es el eje central de nuestro trabajo

Hasta ahora hemos visto la forma que tiene la ecuación de balance de momento para los casos particulares del vacío, fluidos y sólidos, ademas de las densidades de fuerza en dichos casos. Por otra parte ya que a simple vista la ecuación (5.13) no parece ser equivalente a los balances (4.28) y (4.44), entonces podríamos cuestionarnos ¿que medio describe esta nueva ecuación de balance de momento? y más aún ¿tienen alguna relación los balances usuales con esta nueva ecuación de balance?

Si mostramos que las densidades de fuerza $\vec{f}_{H}$ y $f_{i(s)}$ están contenidas en esta nueva ecuación de balance de momento, mostraremos así que (5.13) es un balance más general que los escritos usualmente. Esto es en esencia la motivación principal de este trabajo, mostrar la solidez de esta nueva alternativa para la ecuación de balance de momento en medios materiales.

En esta sección se obtuvo la ecuación de balance de momento mediante un camino alterno, la intención de este trabajo, no es únicamente mostrar este método, también estamos interesados en mostrar la generalidad de esta ecuación, esto es, mostrar que densidades de fuerza están contenidas en nuestra ecuación, es decir mostrar que $\vec{f}_{H}$ y $f_{i(s)}$ están contenidas en nuestra expresión. Para mostrar la validez de esta ecuación de balance momento y densidad de fuerza, analizamos como se relacionan ambas deducciones, mostrando que la densidad de fuerza de Helmholtz, aunque no lo parezca a simple vista, está contenida en nuestra ecuación de balance.

\subsection{Balance de momento electromagnético en me- dios materiales fluidos. La densidad de fuerza de Helmholtz}

Para continuar consideramos un caso particular del balance (5.13), como lo es el caso electrostático, con esta consideración podemos escribir la ecuación (5.13) como

$$
\nabla \cdot\left[\vec{D} \vec{E}-\frac{1}{2}(\vec{D} \cdot \vec{E}) \mathbf{I}\right]=\rho \vec{E}+\frac{1}{2}[(\nabla \vec{E}) \cdot \vec{D}-(\nabla \vec{D}) \cdot \vec{E}]
$$

la idea ahora es mostrar, si es que es posible, que esta ecuación de balance (5.14) contiene a la densidad de fuerza de Helmholtz, para ello proponemos 2 métodos. 


\subsubsection{Deducción alternativa. Primer método}

Para mostrar el alcance de la ecuación de balance (5.14), usamos las relaciones constitutivas. Las relaciones constitutivas además de contener información característica del medio, nos permiten reinterpretar términos de esta ecuación.

Cuando consideramos que el medio es un fluido la ecuación constitutiva es de la siguiente forma

$$
\vec{D}=\epsilon_{0} \epsilon_{r} \vec{E}
$$

y en términos de la susceptibilidad eléctrica

$$
\vec{D}=\epsilon_{0}\left(1+\chi_{e}\right) \vec{E},
$$

sustituimos las ecuaciones (5.15) y (5.16) en el balance (5.14) y obtenemos

$$
\nabla \cdot\left\{\vec{D} \vec{E}-\frac{1}{2} \epsilon_{0}\left[\left(1+\chi_{e}\right) \vec{E} \cdot \vec{E}\right] \mathbf{I}\right\}=\rho \vec{E}-\frac{1}{2} \epsilon_{0}\left[\left(\nabla \epsilon_{r} \vec{E}\right) \cdot \vec{E}-(\nabla \vec{E}) \cdot \epsilon_{r} \vec{E}\right],
$$

al reescribir esta ecuación de balance obtenemos

$$
\nabla \cdot\left[\vec{D} \vec{E}-\frac{1}{2} \epsilon_{0}(\vec{E} \cdot \vec{E}) \mathbf{I}\right]=\rho \vec{E}-\frac{1}{2} \epsilon_{0}\left(\nabla \epsilon_{r}\right) E^{2}+\frac{1}{2} \epsilon_{0} \nabla\left(\chi_{e} E^{2}\right)
$$

Si analizamos el balance (5.18), podemos darnos cuenta que el miembro derecho de esta ecuación es una densidad de fuerza para fluidos, es decir

$$
\overrightarrow{f_{f}}=\rho \vec{E}-\frac{1}{2} \epsilon_{0}\left(\nabla \epsilon_{r}\right) E^{2}+\frac{1}{2} \epsilon_{0} \nabla\left(\chi_{e} E^{2}\right),
$$

la cual es de una estructura similar a la densidad de fuerza de Helmholtz $\vec{f}_{H}$, pero a pesar de ser similares las densidades de fuerza $\vec{f}_{H}$ y $\vec{f}_{f}$ no son las mismas, por ello consideramos que para gases y algunos fluidos podemos utilizar la relación de Clausius - Mossotti, la cual contiene el vínculo entre $\chi_{e}$ y $\rho_{m}$ de la siguiente forma

$$
\chi_{e}=\rho_{m} \frac{\partial \epsilon_{r}}{\partial \rho_{m}},
$$

si utilizamos esta expresión en la ecuación (5.18) obtenemos

$$
\nabla \cdot\left[\vec{D} \vec{E}-\frac{1}{2} \epsilon_{0}(\vec{E} \cdot \vec{E}) \mathbf{I}\right]=\rho \vec{E}-\frac{1}{2} \epsilon_{0}(\nabla \epsilon) E^{2}+\frac{1}{2} \epsilon_{0} \nabla\left(\rho_{m} \frac{\partial \epsilon_{r}}{\partial \rho_{m}} E^{2}\right),
$$

es claro que el término de la derecha de esta ecuación es la densidad fuerza de Helmholtz

$$
\overrightarrow{f_{H}}=\rho \vec{E}-\frac{1}{2} \epsilon_{0}\left(\nabla \epsilon_{r}\right) E^{2}+\frac{1}{2} \epsilon_{0} \nabla\left(\rho_{m} \frac{\partial \epsilon_{r}}{\partial \rho_{m}} E^{2}\right) .
$$

Con este desarrollo, hemos mostrado que la densidad de fuerza de Helmholtz se encuentra contenida de manera natural como un caso particular en nuestra ecuación de balance de momento (5.13), sin necesidad de añadir términos extras al tensor de Maxwell [9]. 


\subsubsection{Deducción alternativa. Segundo método}

Ahora con el fin de mostrar que la ecuación de balance (5.14) es consistente, mostramos que la densidad de fuerza de Helmholtz pude también obtenerse del miembro derecho de esta ecuación.

Con el fin de centrar nuestra atención en el miembro derecho de esta ecuación, escribimos al tensor electrostático de Maxwell como

$$
\mathbf{T}_{E}=\vec{D} \vec{E}-\frac{1}{2}(\vec{D} \cdot \vec{E}) \mathbf{I}
$$

así pues, podemos escribir la ecuación (5.14) como

$$
\nabla \cdot \mathbf{T}_{E}=\rho \vec{E}+\frac{1}{2}[(\nabla \vec{E}) \cdot \vec{D}-(\nabla \vec{D}) \cdot \vec{E}]
$$

considerando que el medio es un fluido y con ayuda de las ecuaciones constitutivas (5.15) y (5.16), podemos reescribir los siguientes términos

$$
\begin{aligned}
(\nabla \vec{D}) \cdot \vec{E} & =\left(\nabla \epsilon_{0} \epsilon_{r} \vec{E}\right) \cdot \vec{E} \\
& =\epsilon_{0}\left[\left(\nabla \epsilon_{r}\right) \vec{E}+\epsilon_{r}(\nabla \vec{E})\right] \cdot \vec{E} \\
& =\epsilon_{0}\left(\nabla \epsilon_{r}\right) E^{2}+(\nabla \vec{E}) \cdot \vec{D}
\end{aligned}
$$

$\mathrm{y}$

$$
\begin{aligned}
(\nabla \vec{E}) \cdot \vec{D} & =\nabla(\vec{E} \cdot \vec{D})-\vec{E} \cdot \nabla \vec{D} \\
& =\nabla\left[\vec{E} \cdot \epsilon_{0}\left(1+\chi_{e}\right) \vec{E}\right]-\vec{E} \cdot \nabla \vec{D} \\
& =\epsilon_{0} \nabla\left(E^{2}+\chi_{e} E^{2}\right)-\vec{E} \cdot \nabla \vec{D} \\
& =\epsilon_{0} \nabla\left(E^{2}\right)+\epsilon_{0} \nabla\left(\chi_{e} E^{2}\right)-\vec{E} \cdot \nabla \vec{D}
\end{aligned}
$$

Sustituyendo las expresiones (5.25) y (5.26) en la ecuación de balance (5.24) resulta

$$
\begin{aligned}
\nabla \cdot \mathbf{T}_{E} & =\rho \vec{E}+\frac{1}{2}\left[\epsilon_{0} \nabla\left(E^{2}\right)+\epsilon_{0} \nabla\left(\chi_{e} E^{2}\right)-\vec{E} \cdot \nabla \vec{D}-\epsilon_{0}\left(\nabla \epsilon_{r}\right) E^{2}-(\nabla \vec{E}) \cdot \vec{D}\right] \\
& =\rho \vec{E}-\frac{1}{2} \epsilon_{0}\left(\nabla \epsilon_{r}\right) E^{2}+\frac{1}{2} \epsilon_{0} \nabla\left(\chi_{e} E^{2}\right)-\frac{1}{2} \nabla\left(\vec{D} \cdot \vec{E}-\epsilon_{0} E^{2}\right)
\end{aligned}
$$

Considerando que

$$
\nabla\left(\vec{D} \cdot \vec{E}-\epsilon_{0} E^{2}\right)=\nabla \cdot \mathbf{I}\left(\vec{D} \cdot \vec{E}-\epsilon_{0} E^{2}\right)
$$

y la relación

$$
\vec{D}=\epsilon_{0} \vec{E}+\epsilon_{0} \vec{P}
$$

podemos escribir la ecuación de balance (5.27) como 


$$
\nabla \cdot\left[\mathbf{T}_{E}+\frac{1}{2} \mathbf{I}(\vec{P} \cdot \vec{E})\right]=\rho \vec{E}-\frac{1}{2} \epsilon_{0} E^{2} \nabla \epsilon_{r}+\frac{1}{2} \epsilon_{0} \nabla\left(\chi_{e} E^{2}\right),
$$

análogamente como lo consideramos en la sección anterior, si consideramos un medio que cumple con la ecuación de Clausius- Mossottie, es decir

$$
\chi_{e}=\rho_{m} \frac{\partial \epsilon_{r}}{\partial \rho_{m}}
$$

podemos ver claramente que el miembro derecho de la ecuación de balance (5.30) corresponde a la densidad de fuerza de Hemlholtz

$$
\nabla \cdot\left[\mathbf{T}_{E}+\frac{1}{2} \mathbf{I}(\vec{P} \cdot \vec{E})\right]=\rho \vec{E}-\frac{1}{2} \epsilon_{0} E^{2} \nabla \epsilon_{r}+\frac{1}{2} \epsilon_{0} \nabla\left(\rho_{m} \frac{\partial \epsilon_{r}}{\partial \rho_{m}} E^{2}\right)
$$

Con esta deducción mostramos que la ecuación de balance de momento electromagnético (5.14) es una ecuación consistente. Considerando que Campos et al en [16], usando el miembro izquierdo de esta ecuación, mostraron que la densidad de fuerza de Hemlholtz estaba contenida en la ecuación de balance de momento, ahora en este trabajo hemos demostrado que es posible también deducir la densidad de fuerza de Hemlholtz utilizando el miembro derecho de la ecuación de balance de momento electromagnético. Queda establecida la consistencia de la ecuación (5.14). Ahora, dado que nuestra ecuación contiene al menos a la densidad de fuerza $\vec{f}_{(H)}$ como caso particular, podemos preguntarnos ¿está contenida la densidad de fuerza de un sólido $f_{i(s)}$ en nuestra ecuación de balance de momento?

\subsection{Balance de momento electromagnético en me- dios materiales sólidos, deducciones alternativas}

Esta sección es la parte medular de nuestro trabajo, aquí demostramos que la densidad de fuerza sobre un sólido, puede ser deducida directamente de la ecuación de balance de momento electromagnético y densidad de fuerza. Para exhibir esto, presentamos dos nuevos métodos, que aun siendo distintos, se enfocan en mostrar el alcance de la ecuación de balance (5.14) y las ecuaciones constitutivas.

\subsubsection{Deducción alternativa. Primer método}

En este primer método partimos de la ecuación de balance (5.14), ahora analizamos un medio cuyas propiedades estén descritas, análogamente como considera Robison, mediante una relación constitutiva de la siguiente forma

$$
D_{j}=\epsilon_{0} \epsilon_{j k} E_{k}
$$


es decir un sólido.

Escribiendo la ecuación de balance (5.14) en componentes resulta

$$
\frac{\partial}{\partial r_{j}}\left[D_{i} E_{j}-\frac{1}{2}\left(D_{l} E_{l}\right) \delta_{i j}\right]=\rho E_{i}+\frac{1}{2}\left[\left(\frac{\partial E_{j}}{\partial r_{i}}\right) D_{j}-\left(\frac{\partial D_{j}}{\partial r_{i}}\right) E_{j}\right]
$$

sustituyendo la relación constitutiva para un sólido en el miembro de la derecha de la ecuación tenemos

$$
\begin{aligned}
\frac{\partial}{\partial r_{j}}\left[D_{i} E_{j}-\frac{1}{2}\left(D_{l} E_{l}\right) \delta_{i j}\right] & =\rho E_{i}-\frac{1}{2} \epsilon_{0}\left[\left(\frac{\partial \epsilon_{j k} E_{k}}{\partial r_{i}}\right) E_{j}-\left(\frac{\partial E_{j}}{\partial r_{i}}\right) \epsilon_{j k} E_{k}\right] \\
& =\rho E_{i}-\frac{1}{2} E_{k} E_{j} \epsilon_{0} \frac{\partial \epsilon_{j k}}{\partial r_{i}}-\frac{1}{2} \epsilon_{0} \epsilon_{j k}\left[E_{j}\left(\frac{\partial E_{k}}{\partial r_{i}}\right)-\left(\frac{\partial E_{j}}{\partial r_{i}}\right) E_{k}\right] \\
& =\rho E_{i}-\frac{1}{2} \epsilon_{0} E_{k} E_{j} \frac{\partial \epsilon_{j k}}{\partial r_{i}}-\frac{1}{2} \epsilon_{0}\left[E_{j} \epsilon_{j k}\left(\frac{\partial E_{k}}{\partial r_{i}}\right)-\left(\frac{\partial E_{j}}{\partial r_{i}}\right) \epsilon_{j k} E_{k}\right] \\
& =\rho E_{i}-\frac{1}{2} \epsilon_{0} E_{k} E_{j} \frac{\partial \epsilon_{j k}}{\partial r_{i}}-\frac{1}{2}\left[D_{k}\left(\frac{\partial E_{k}}{\partial r_{i}}\right)-\left(\frac{\partial E_{j}}{\partial r_{i}}\right) D_{j}\right] .(5.35)
\end{aligned}
$$

Analizando el último término de la derecha, si $j \rightarrow k$

$$
\left[D_{k}\left(\frac{\partial E_{k}}{\partial r_{i}}\right)-\left(\frac{\partial E_{j}}{\partial r_{i}}\right) D_{j}\right]=\left[D_{k}\left(\frac{\partial E_{k}}{\partial r_{i}}\right)-\left(\frac{\partial E_{k}}{\partial r_{i}}\right) D_{k}\right]=0
$$

entonces podemos escribir la ecuación (5.35) como

$$
\frac{\partial}{\partial r_{j}}\left[D_{i} E_{j}-\frac{1}{2}\left(D_{l} E_{l}\right) \delta_{i j}\right]=\rho E_{i}-\frac{1}{2} \epsilon_{0} E_{k} E_{j}\left(\frac{\partial \epsilon_{j k}}{\partial r_{i}}\right) .
$$

Considerando que la relación constitutiva (5.33) puede ser escrita en términos de la susceptibilidad eléctrica de la siguiente forma

$$
D_{l}=\epsilon_{0}\left(\delta_{k l}+\chi_{l k}\right) E_{k}
$$

y sustituyendo en el miembro izquierdo de la ecuación (5.36) tenemos

$$
\frac{\partial}{\partial r_{j}}\left[D_{i} E_{j}-\frac{1}{2} \epsilon_{0}\left(\delta_{k l}+\chi_{l k}\right) E_{k} E_{l} \delta_{i j}\right]=\rho E_{i}-\frac{1}{2} \epsilon_{0} E_{k} E_{j}\left(\frac{\partial \epsilon_{j k}}{\partial r_{i}}\right),
$$

realizando los productos reescribimos esta ecuación como

$$
\frac{\partial}{\partial r_{j}}\left(D_{i} E_{j}-\frac{1}{2} \epsilon_{0} E_{k} E_{l} \delta_{k l} \delta_{i j}\right)=\rho E_{i}-\frac{1}{2} \epsilon_{0} E_{k} E_{j}\left(\frac{\partial \epsilon_{j k}}{\partial r_{i}}\right)+\frac{\partial}{\partial r_{j}}\left(\frac{1}{2} \epsilon_{0} E_{k} E_{l} \chi_{l k} \delta_{i j}\right) .
$$

Ahora nos interesa analizar el término $\chi_{l k} \delta_{i j}$, el cual es un tensor de cuarto rango que claramente es simétrico en los índices $i$ y $j$ debido a las propiedades de $\delta$ y 
simétrico en los índices $l$ y $k$ considerando que $\chi_{l k}$ es un tensor simétrico. Entonces si definimos

$$
\chi_{l k} \delta_{i j} \equiv-\alpha_{l k i j}
$$

donde $\alpha_{l k i j}$ es el tensor de cuarto rango que tiene las siguientes características $\alpha_{l k i j}=$ $\alpha_{k l i j}=\alpha_{l k j i}$, analizando esta características de $\alpha$ nos damos cuenta que coinciden perfectamente con las características del tensor $a_{l m}^{j k}$ propuesto por Stratton [1] para describir las propiedades elásticas del medio, así podemos establecer la igualdad de $\alpha_{l k i j}$ y $a_{l m}^{j k}$. Escribiendo (5.39) en términos de $\alpha_{l k i j}$, tenemos

$$
\frac{\partial}{\partial r_{j}}\left(D_{i} E_{j}-\frac{1}{2} \epsilon_{0} E_{l} E_{l} \delta_{i j}\right)=\rho E_{i}-\frac{1}{2} \epsilon_{0} E_{k} E_{j}\left(\frac{\partial \epsilon_{j k}}{\partial r_{i}}\right)+\frac{\partial}{\partial r_{j}}\left(-\frac{1}{2} \epsilon_{0} E_{k} E_{l} \alpha_{l k i j}\right),
$$

de esta ecuación podemos identificar la densidad de fuerza para un sólido $f_{i(s)}$ como

$$
f_{(s) i}=\rho E_{i}-\frac{1}{2} \epsilon_{0} E_{k} E_{j}\left(\frac{\partial \epsilon_{j k}}{\partial r_{i}}\right)+\frac{\partial}{\partial r_{j}}\left(-\frac{1}{2} \epsilon_{0} E_{k} E_{l} \alpha_{l k i j}\right) .
$$

\section{Mismo enfoque. Otro camino}

Con este mismo enfoque, de manera análoga que para fluidos, es posible obtener la densidad de fuerza sobre sólidos elásticos directamente del miembro derecho de la ecuación de balance de momento (5.13). Considerando unicamente campos electrostáticos podemos escribir

$$
\frac{\partial}{\partial r_{j}}\left(T_{i j}^{E}\right)=\rho E_{i}+\frac{1}{2}\left[\left(\frac{\partial E_{j}}{\partial r_{i}}\right) D_{j}-\left(\frac{\partial D_{j}}{\partial r_{i}}\right) E_{j}\right]
$$

sustituyendo las ecuaciones constitutivas

$$
D_{j}=\epsilon_{0} \epsilon_{j k} E_{k}
$$

$\mathrm{y}$

$$
D_{l}=\epsilon_{0}\left(\delta_{k l}+\chi_{l k}^{e}\right) E_{k},
$$

ademas considerando las igualdades

$$
\left(\frac{\partial D_{j}}{\partial r_{i}}\right) E_{j}=\epsilon_{0}\left(\frac{\partial \epsilon_{j k}}{\partial r_{i}}\right) E_{k} E_{j}+\left(\frac{\partial E_{k}}{\partial r_{i}}\right) D_{k}
$$

y

$$
\begin{aligned}
\left(\frac{\partial E_{j}}{\partial r_{i}}\right) D_{j} & =\frac{\partial}{\partial r_{j}}\left(E_{l} D_{l} \delta_{i j}\right)-E_{l} \frac{\partial}{\partial r_{j}}\left(D_{l} \delta_{i j}\right) \\
& =\frac{\partial}{\partial r_{j}}\left[E_{l} \epsilon_{0}\left(\delta_{k l}+\chi_{l k}^{e}\right) E_{k} \delta_{i j}\right]-E_{l} \frac{\partial}{\partial r_{j}}\left(D_{l} \delta_{i j}\right) \\
& =\frac{\partial}{\partial r_{j}}\left(\epsilon_{0} E^{2} \delta_{i j}\right)+\frac{\partial}{\partial r_{j}}\left(\epsilon_{0} \chi_{l k}^{e} \delta_{i j} E_{l} E_{k}\right)-E_{l} \frac{\partial}{\partial r_{j}}\left(D_{l} \delta_{i j}\right)
\end{aligned}
$$


podemos escribir la ecuación de balance como

$$
\begin{aligned}
\frac{\partial}{\partial r_{j}}\left(T_{i j}^{E}\right) & =-\frac{1}{2}\left[\epsilon_{0}\left(\frac{\partial \epsilon_{j k}}{\partial r_{i}}\right) E_{k} E_{j}+\left(\frac{\partial E_{k}}{\partial r_{i}}\right) D_{k}\right] \\
& +\rho E_{i}+\frac{1}{2}\left[\frac{\partial}{\partial r_{j}}\left(\epsilon_{0} E^{2} \delta_{i j}\right)+\frac{\partial}{\partial r_{j}}\left(\epsilon_{0} \chi_{l k}^{e} \delta_{i j} E_{l} E_{k}\right)-E_{l} \frac{\partial}{\partial r_{j}}\left(D_{l} \delta_{i j}\right)\right]
\end{aligned}
$$

reescribiendo los términos tenemos que

$$
\begin{gathered}
\frac{\partial}{\partial r_{j}}\left(T_{i j}^{E}\right)=\rho E_{i}+\frac{1}{2} \frac{\partial}{\partial r_{j}}\left(\epsilon_{0} \chi_{l k}^{e} \delta_{i j} E_{l} E_{k}\right)-\frac{1}{2} \epsilon_{0}\left(\frac{\partial \epsilon_{j k}}{\partial r_{i}}\right) E_{k} E_{j} \\
+\frac{1}{2}\left[\frac{\partial}{\partial r_{j}}\left(\epsilon_{0} E^{2} \delta_{i j}\right)-\left(\frac{\partial E_{k}}{\partial r_{i}}\right) D_{k}-E_{l} \frac{\partial}{\partial r_{j}}\left(D_{l} \delta_{i j}\right)\right]
\end{gathered}
$$

ahora agrupando términos obtenemos

$$
\begin{aligned}
\frac{\partial}{\partial r_{j}}\left(T_{i j}^{E}\right) & =\frac{1}{2} \frac{\partial}{\partial r_{j}}\left(\epsilon_{0} E^{2} \delta_{i j}-E_{k} D_{k} \delta_{i j}\right) \\
+\rho E_{i} & +\frac{1}{2} \frac{\partial}{\partial r_{j}}\left(\epsilon_{0} \chi_{l k}^{e} \delta_{i j} E_{l} E_{k}\right)-\frac{1}{2} \epsilon_{0}\left(\frac{\partial \epsilon_{j k}}{\partial r_{i}}\right) E_{k} E_{j}
\end{aligned}
$$

Con esto podemos escribir la ecuación de la balance (5.42) como

$$
\frac{\partial}{\partial r_{j}}\left[T_{i j}^{E}+\frac{1}{2}\left(E_{k} D_{k}-\epsilon_{0} E^{2}\right) \delta_{i j}\right]=\rho E_{i}+\frac{1}{2} \frac{\partial}{\partial r_{j}}\left(\epsilon_{0} \chi_{l k}^{e} \delta_{i j} E_{l} E_{k}\right)-\frac{1}{2} \epsilon_{0}\left(\frac{\partial \epsilon_{j k}}{\partial r_{i}}\right) E_{k} E_{j}
$$

Definiendo el tensor

$$
\alpha_{i j l k}=-\chi_{l k}^{e} \delta_{i j}
$$

y usando la relación

$$
D_{k}=\epsilon_{0}\left(E_{k}+P_{k}\right)
$$

como hemos discutido en la sección capitulo anterior, con esta identidad podemos escribir

$$
\frac{\partial}{\partial r_{j}}\left[T_{i j}^{E}+\frac{1}{2}\left(P_{k} E_{k}\right) \delta_{i j}\right]=\rho E_{i}+\frac{1}{2} \frac{\partial}{\partial r_{j}}\left(-\epsilon_{0} \alpha_{i j l k} E_{l} E_{k}\right)-\frac{1}{2} \epsilon_{0}\left(\frac{\partial \epsilon_{j k}}{\partial r_{i}}\right) E_{k} E_{j} .
$$

Con esto hemos mostrado que efectivamente nuestra ecuación (5.14) contiene al menos como casos particulares la densidad de fuerza $f_{i(s)}$ y $\vec{f}_{H}$, lo que nos deja ver de manera clara que el balance propuesto, es una alternativa más general que los propuestos usualmente, puesto que es posible describir tanto a sólido como a fluidos. 
5.2 .

\subsubsection{Deducción alternativa. Segundo método.}

Por otra parte es posible mostrar, siguiendo otro camino, que la densidad de fuerza para un sólido $f_{i(s)}$ está contenida en la ecuación de balance momento electromagnético (5.14), cuando se analiza un caso particular. Para presentar esta nueva deducción, partimos de la ecuación de balance de momento para el caso electrostático, el miembro izquierdo de esta ecuación puede escribirse como

$$
\nabla \cdot \mathbf{T}_{E}=\nabla \cdot(\vec{D} \vec{E})-\frac{1}{2} \nabla(\vec{D} \cdot \vec{E})
$$

Aquí, análogamente como lo consideran Stratton y Robinson, consideramos que para un sólido la permitividad depende de la posición $\vec{r}$ y del tensor de tensiones $S_{l m}$

$$
\epsilon \equiv \epsilon\left(\vec{r}, S_{l m}\right)
$$

Motivados por esta dependencia de la permitividad, redefinimos la forma del gradiente, esto es

$$
\nabla \rightarrow\left(\frac{\partial}{\partial r_{i}}+\frac{\partial S_{l m}}{\partial r_{i}} \frac{\partial}{\partial S_{l m}}\right)
$$

Usando esta definición de gradiente en la ecuación (5.54), obtenemos

$$
\begin{aligned}
\nabla \cdot \mathbf{T}_{E} & =\left(\frac{\partial}{\partial r_{i}}+\frac{\partial S_{l m}}{\partial r_{i}} \frac{\partial}{\partial S_{l m}}\right)\left(D_{i} E_{j}\right)-\frac{1}{2}\left(\frac{\partial}{\partial r_{j}}+\frac{\partial S_{l m}}{\partial r_{j}} \frac{\partial}{\partial S_{l m}}\right)\left(D_{i} E_{i}\right) \\
& =\left[\frac{\partial}{\partial r_{i}}\left(D_{i} E_{j}\right)-\frac{1}{2} \frac{\partial}{\partial r_{j}}\left(D_{i} E_{i}\right)\right]+\left[\frac{\partial S_{l m}}{\partial r_{i}} \frac{\partial}{\partial S_{l m}}\left(D_{i} E_{j}\right)-\frac{1}{2} \frac{\partial S_{l m}}{\partial r_{j}} \frac{\partial}{\partial S_{l m}}\left(D_{i} E_{i}\right)\right] .
\end{aligned}
$$

Sea

$$
A_{j} \equiv \frac{\partial}{\partial r_{i}}\left(D_{i} E_{j}\right)-\frac{1}{2} \frac{\partial}{\partial r_{j}}\left(D_{i} E_{i}\right)
$$

y

$$
B_{j} \equiv \frac{\partial S_{l m}}{\partial r_{i}} \frac{\partial}{\partial S_{l m}}\left(D_{i} E_{j}\right)-\frac{1}{2} \frac{\partial S_{l m}}{\partial r_{j}} \frac{\partial}{\partial S_{l m}}\left(D_{i} E_{i}\right),
$$

entonces podemos escribir

$$
\nabla \cdot \mathbf{T}_{E}=A_{j}+B_{j}
$$

Ahora analizo el término $A_{j}$

$$
\begin{aligned}
A_{j} & =\frac{\partial}{\partial r_{i}}\left(D_{i} E_{j}\right)-\frac{1}{2} \frac{\partial}{\partial r_{j}}\left(D_{i} E_{i}\right) \\
& =D_{i}\left(\frac{\partial E_{j}}{\partial r_{i}}\right)+E_{j}\left(\frac{\partial D_{i}}{\partial r_{i}}\right)-\frac{1}{2}\left[D_{i}\left(\frac{\partial E_{i}}{\partial r_{j}}\right)+E_{i}\left(\frac{\partial D_{i}}{\partial r_{j}}\right)\right] .
\end{aligned}
$$

Si usamos que $D_{i}=\epsilon_{0} \epsilon_{i k} E_{k}$, tenemos que 


$$
\begin{aligned}
A_{j} & =D_{i}\left(\frac{\partial E_{j}}{\partial r_{i}}\right)+E_{j}\left(\frac{\partial D_{i}}{\partial r_{i}}\right)-\frac{1}{2}\left[D_{i}\left(\frac{\partial E_{i}}{\partial r_{j}}\right)+\epsilon_{0} E_{i}\left(\frac{\partial \epsilon_{i k} E_{k}}{\partial r_{j}}\right)\right] \\
& =D_{i}\left(\frac{\partial E_{j}}{\partial r_{i}}\right)+E_{j}\left(\frac{\partial D_{i}}{\partial r_{i}}\right)-\frac{1}{2}\left[2 D_{i}\left(\frac{\partial E_{i}}{\partial r_{j}}\right)+\epsilon_{0} E_{i} E_{k}\left(\frac{\partial \epsilon_{i k}}{\partial r_{j}}\right)\right] \\
& =E_{j}\left(\frac{\partial D_{i}}{\partial r_{i}}\right)-\frac{1}{2} \epsilon_{0} E_{i} E_{k}\left(\frac{\partial \epsilon_{i k}}{\partial r_{j}}\right)+D_{i}\left[\left(\frac{\partial E_{j}}{\partial r_{i}}\right)-\left(\frac{\partial E_{i}}{\partial r_{j}}\right)\right]
\end{aligned}
$$

dado que estamos en un caso electrostático

$$
\nabla \times \vec{E}=0
$$

por lo tanto

$$
\left(\frac{\partial E_{j}}{\partial r_{i}}\right)=\left(\frac{\partial E_{i}}{\partial r_{j}}\right)
$$

así podemos escribir finalmente el vector $A_{j}$ como

$$
A_{j}=E_{j}\left(\frac{\partial D_{i}}{\partial r_{i}}\right)-\frac{1}{2} \epsilon_{0} E_{i} E_{k}\left(\frac{\partial \epsilon_{i k}}{\partial r_{j}}\right) .
$$

Tomando en cuenta la ecuación (5.55) podemos escribir que

$$
D_{i}=D\left(r_{i}, S_{l m}\right)
$$

entonces analizando el vector $B_{j}$ podemos escribir

$$
\begin{aligned}
B_{j} & =\frac{\partial S_{l m}}{\partial r_{i}} \frac{\partial}{\partial S_{l m}}\left(D_{i} E_{j}\right)-\frac{1}{2} \frac{\partial S_{l m}}{\partial r_{j}} \frac{\partial}{\partial S_{l m}}\left(D_{i} E_{i}\right) \\
& =E_{j} \frac{\partial S_{l m}}{\partial r_{i}}\left(\frac{\partial D_{i}}{\partial S_{l m}}\right)-\frac{1}{2} E_{i} \frac{\partial S_{l m}}{\partial r_{j}}\left(\frac{\partial D_{i}}{\partial S_{l m}}\right) \\
& =E_{j} \frac{\partial S_{l m}}{\partial r_{i}}\left(\frac{\partial D_{i}}{\partial S_{l m}}\right)-\frac{1}{2} \epsilon_{0} E_{i} E_{k} \frac{\partial S_{l m}}{\partial r_{j}}\left(\frac{\partial \epsilon_{i k}}{\partial S_{l m}}\right) .
\end{aligned}
$$

Sustituyendo las ecuaciones (5.60) y (5.62) en la expresión (5.57)

$$
\begin{aligned}
\nabla \cdot \mathbf{T}_{E} & =E_{j}\left(\frac{\partial D_{i}}{\partial r_{i}}\right)-\frac{1}{2} \epsilon_{0} E_{i} E_{k}\left(\frac{\partial \epsilon_{i k}}{\partial r_{j}}\right)+E_{j} \frac{\partial S_{l m}}{\partial r_{i}}\left(\frac{\partial D_{i}}{\partial S_{l m}}\right)-\frac{1}{2} \epsilon_{0} E_{i} E_{k} \frac{\partial S_{l m}}{\partial r_{j}}\left(\frac{\partial \epsilon_{i k}}{\partial S_{l m}}\right) \\
& =E_{j}\left(\frac{\partial D_{i}}{\partial r_{i}}+\frac{\partial S_{l m}}{\partial r_{i}} \frac{\partial D_{i}}{\partial S_{l m}}\right)-\frac{1}{2} \epsilon_{0} E_{i} E_{k}\left(\frac{\partial \epsilon_{i k}}{\partial r_{j}}+\frac{\partial S_{l m}}{\partial r_{j}} \frac{\partial \epsilon_{i k}}{\partial S_{l m}}\right) .
\end{aligned}
$$


5.2 .

Por otra parte, usando la definición de gradiente podemos escribir

$$
\left(\frac{\partial D_{i}}{\partial r_{i}}+\frac{\partial S_{l m}}{\partial r_{i}} \frac{\partial D_{i}}{\partial S_{l m}}\right)=\nabla \cdot \vec{D}=\rho,
$$

y definiendo

$$
\alpha_{i k l m}=\frac{\partial \epsilon_{i k}}{\partial S_{l m}},
$$

podemos escribir la ecuación (5.63) como

$$
\nabla \cdot \mathbf{T}_{E}=\rho E_{j}-\frac{1}{2} \epsilon_{0} E_{i} E_{k} \frac{\partial \epsilon_{i k}}{\partial r_{j}}-\frac{1}{2} \epsilon_{0} E_{i} E_{k} \frac{\partial S_{l m}}{\partial r_{j}} \alpha_{i k l m} .
$$

Hasta ahora, en la ecuación (5.66), no es evidente la expresión para la densidad de fuerza $f_{i(s)}$, así para continuar analizamos el término

$$
\frac{\partial S_{l m}}{\partial r_{j}}
$$

Recordando que

$$
S_{l m}=\frac{\partial u_{l}}{\partial r_{m}}
$$

podemos escribir

$$
\frac{\partial S_{l m}}{\partial r_{j}}=\frac{\partial}{\partial r_{j}}\left(\frac{\partial u_{l}}{\partial r_{m}}\right)=\frac{\partial}{\partial r_{m}}\left(\frac{\partial u_{l}}{\partial r_{j}}\right)=\frac{\partial S_{l j}}{\partial r_{m}}
$$

esta igualdad nos permite escribir

$$
\frac{1}{2} \epsilon_{0} E_{i} E_{k} \frac{\partial S_{l m}}{\partial r_{j}} \alpha_{i k l m}=\frac{1}{2} \epsilon_{0} E_{i} E_{k} \frac{\partial S_{l j}}{\partial r_{m}} \alpha_{i k l m} .
$$

Si definimos

$$
C_{m j}=\frac{1}{2} \epsilon_{0} E_{i} E_{k} \alpha_{i k l m} S_{l j},
$$

podemos escribir (5.69) como

$$
\frac{1}{2} \epsilon_{0} E_{i} E_{k} \frac{\partial S_{l j}}{\partial r_{m}} \alpha_{i k l m}=\frac{\partial}{\partial r_{m}} C_{m j}-S_{l j} \frac{\partial}{\partial r_{m}}\left(\frac{1}{2} \epsilon_{0} E_{i} E_{k} \alpha_{i k l m}\right) .
$$

Sustituyendo (5.71) en (5.66) e integrando ambos lados de la ecuación tenemos

$$
\int \nabla \cdot \mathbf{T}_{E} d V=\int\left[\rho E_{j}-\frac{1}{2} \epsilon_{0} E_{i} E_{k} \frac{\partial \epsilon_{i k}}{\partial r_{j}}-\frac{\partial}{\partial r_{m}} C_{m j}+S_{l j} \frac{\partial}{\partial r_{m}}\left(\frac{1}{2} \epsilon_{0} E_{i} E_{k} \alpha_{i k l m}\right)\right] d V
$$


usando el teorema de Gauss y escogiendo una superficie adecuada podemos anular el término

$$
\int \frac{\partial}{\partial r_{m}} C_{m j} d V
$$

con lo que finalmente podemos escribir nuestra ecuación de balance de momento de la siguiente manera

$$
\nabla \cdot \mathbf{T}_{E}=\rho E_{j}-\frac{1}{2} \epsilon_{0} E_{i} E_{k} \frac{\partial \epsilon_{i k}}{\partial r_{j}}+S_{l j} \frac{\partial}{\partial r_{m}}\left(\frac{1}{2} \epsilon_{0} E_{i} E_{k} \alpha_{i k l m}\right)
$$

Hasta este punto de nuestro desarrollo, no hemos hecho consideraciones que limiten la generalidad de esta ecuación, para seguir con este análisis es necesario hacer una suposición. Consideramos que

$$
S_{l j}=\frac{\partial u_{l}}{\partial r_{j}}=A \delta_{l j} .
$$

Es posible hacer esta consideración cuando la deformación del material es igual en todas las direcciones, donde $A$ es una constante que depende del medio.

Sustituyendo la ecuación (5.74) en la ecuación (5.73) obtenemos

$$
\begin{aligned}
\nabla \cdot \mathbf{T}_{E} & =\rho E_{j}-\frac{1}{2} \epsilon_{0} E_{i} E_{k} \frac{\partial \epsilon_{i k}}{\partial r_{j}}+\delta_{l j} \frac{\partial}{\partial r_{m}}\left(\frac{1}{2} \epsilon_{0} E_{i} E_{k} \alpha_{i k l m}\right) \\
& =\rho E_{j}-\frac{1}{2} \epsilon_{0} E_{i} E_{k} \frac{\partial \epsilon_{i k}}{\partial r_{j}}+\frac{\partial}{\partial r_{m}}\left(\frac{1}{2} \epsilon_{0} E_{i} E_{k} \alpha_{i k j m}\right) .
\end{aligned}
$$

La ecuación de balance de momento (5.75), es un caso particular de nuestra ecuación de balance de momento (5.14). El miembro derecho de la ecuación (5.75) corresponde a una densidad de fuerza, esta densidad de fuerza es la densidad de fuerza para un sólido $f_{j(s)}$ y coincide con la encontrada por Stratton[1], Robinson[4], entre otros. Con esto mostramos otro método para deducir la densidad de fuerza en un sólido a partir de nuestra ecuación de balance de momento electromagnético (5.14). Este método es un elemento más que apoya la generalidad de nuestro balance.

\subsection{Densidad de fuerza magnética en medios mate- riales}

Para realizar estas deducciones partimos de la ecuación de balance de momento electromagnético obtenida en la sección previa, la forma general de dicha ecuación es

$$
\begin{aligned}
\nabla \cdot \mathbf{T}_{E M} & =\rho \vec{E}+\vec{j} \times \vec{B}+\frac{\partial}{\partial t}(\vec{D} \times \vec{B}) \\
& +\frac{1}{2}[(\nabla \vec{E}) \cdot \vec{D}-(\nabla \vec{D}) \cdot \vec{E}+(\nabla \vec{H}) \cdot \vec{B}-(\nabla \vec{B}) \cdot \vec{H}] .
\end{aligned}
$$


Para continuar consideramos un caso particular de la ecuación (5.13), el caso donde unicamente existen campos magnetostáticos, con esta consideración podemos escribir dicha ecuación como

$$
\nabla \cdot\left[\vec{B} \vec{H}-\frac{1}{2}(\vec{B} \cdot \vec{H})\right]=\vec{j} \times \vec{B}+\frac{1}{2}[(\nabla \vec{H}) \cdot \vec{B}-(\nabla \vec{B}) \cdot \vec{H}]
$$

ahora, análogamente como lo hicimos en el caso electrostático, estamos interesado en mostrar, que, a partir de la ecuación de balance (5.77) podemos deducir las densidades de fuerza magnética en un fluido y en un sólido.

\subsubsection{Densidad de fuerza magnética en fluidos}

Cuando el medio sobre el cual actúan los campos magnetostáticos es un fluido escribimos la ecuación constitutiva que relaciona los campos $\vec{B}$ y $\vec{H}$ de la siguiente forma

$$
\vec{B}=\mu_{0} \mu_{r} \vec{H}
$$

y en términos de la susceptibilidad magnética

$$
\vec{B}=\mu_{0}\left(1+\chi_{m}\right) \vec{H}
$$

Sustituyendo las ecuaciones (5.78) y (5.79) en la ecuación (5.77) obtenemos que

$$
\nabla \cdot\left[\vec{B} \vec{H}-\frac{1}{2} \mu_{0}\left(1+\chi_{m}\right) \vec{H} \cdot \vec{H}\right]=\vec{j} \times \vec{B}-\frac{1}{2} \mu_{0}\left[\left(\nabla \mu_{r} \vec{H}\right) \cdot \vec{H}-(\nabla \vec{H}) \cdot \mu_{r} \vec{H}\right]
$$

reescribiendo esta ecuación resulta

$$
\nabla \cdot\left[\vec{B} \vec{H}-\frac{1}{2} \mu_{0}(\vec{H} \cdot \vec{H})\right]=\vec{j} \times \vec{B}-\frac{1}{2} \mu_{0}\left(\nabla \mu_{r}\right) H^{2}+\frac{1}{2} \mu_{0} \nabla\left(\chi_{m} H^{2}\right),
$$

en donde podemos reconocer el miembro derecho de esta ecuación como una densidad de fuerza, es decir

$$
\overrightarrow{f_{M}}=\vec{j} \times \vec{B}-\frac{1}{2} \mu_{0}\left(\nabla \mu_{r}\right) H^{2}+\frac{1}{2} \mu_{0} \nabla\left(\chi_{m} H^{2}\right)
$$

Hasta este punto hemos considerado un medio cuyas propiedades estén descritas por la ecuación constitutiva (5.88), pero, salvo esta restricción, la densidad de fuerza $\vec{f}_{M}$ es una densidad de fuerza general sobre un fluido.

Debido a que nos interesa hacer una analogía con el caso electrostático es necesario imponer una consideración más al medio. Para medios que satisfacen la relación Clausius - Mossotti, autores como Tamm [9] y Landau y Lifshitz [10] escriben la relación entre la permitividad y la densidad del medio, como

$$
\chi_{e}=\rho_{m} \frac{\partial \epsilon}{\partial \rho_{m}},
$$


En analogía con este caso, escribimos la relación para el caso magnetostático

$$
\chi_{m}=\rho_{m} \frac{\partial \mu_{r}}{\partial \rho_{m}},
$$

sin esta consideración no se pueden comparar densidades de fuerza magnética $\vec{f}_{M}$ y la densidad de fuerza de Helmholtz $\vec{f}_{H}$. Esta relación puede considerarse como una relación análoga a la relación de Clausius - Mossotti, cuando los campos involucrados son magnetostáticos.

Sustituyendo la ecuación (5.93) en la expresión para la densidad de fuerza $\vec{f}_{M}$ obtenemos

$$
\overrightarrow{f_{m}}=\vec{j} \times \vec{B}-\frac{1}{2} \mu_{0}\left(\nabla \mu_{r}\right) H^{2}+\frac{1}{2} \mu_{0} \nabla\left(\rho_{m} \frac{\partial \mu_{r}}{\partial \rho_{m}} H^{2}\right),
$$

donde la densidad de fuerza magnética $\vec{f}_{m}$ es un caso particular de la densidad de fuerza magnética $\vec{f}_{M}$.

Es evidente, ahora, la estructura similar que poseen la densidad de fuerza magnética sobre fluidos $\vec{f}_{m}$ y a densidad de fuerza magnética de Helmholtz $\vec{f}_{H}$.

\section{Mismo Enfoque. Otro Camino}

Dada la simetría que presentan los campos $\vec{E}$ y $\vec{H}$ en la ecuación de balance de momento electromagnético (5.13), podemos mostrar que la densidad de fuerza debida a campos magnetostáticos también puede deducirse directamente del miembro derecho de esta ecuación, cuando consideramos únicamente campos magnetostáticos, es decir

$$
\nabla \cdot\left[\vec{B} \vec{H}-\frac{1}{2}(\vec{B} \cdot \vec{H})\right]=\vec{j} \times \vec{B}+\frac{1}{2}[(\nabla \vec{H}) \cdot \vec{B}-(\nabla \vec{B}) \cdot \vec{H}] .
$$

Considerando que la ecuación constitutiva para los campos $\vec{B}$ y $\vec{H}$

$$
\vec{B}=\mu_{0} \mu_{r} \vec{H}
$$

y en términos de la susceptibilidad magnética

$$
\vec{B}=\mu_{0}\left(1+\chi_{m}\right) \vec{H},
$$

podemos escribir, en completa analogía con la sección anterior, las siguientes igualdades

$$
(\nabla \vec{B}) \cdot \vec{H}=\mu_{0}\left(\nabla \mu_{r}\right) H^{2}+(\nabla \vec{H}) \cdot \vec{B}
$$

y

$$
(\nabla \vec{H}) \cdot \vec{B}=\mu_{0} \nabla\left(H^{2}\right)+\mu_{0} \nabla\left(\chi_{m} H^{2}\right)-\vec{H} \cdot \nabla \vec{B} .
$$

Sustituyendo las expresiones (C.3) y (5.90) en la ecuación de balance (5.86), análogamente con la sección anterior obtenemos

$$
\nabla \cdot\left[\mathbf{T}_{M}+\frac{1}{2} \mathbf{I}(\vec{M} \cdot \vec{H})\right]=\vec{j} \times \vec{B}-\frac{1}{2} \mu_{0} H^{2} \nabla \mu_{r}+\frac{1}{2} \mu_{0} \nabla\left(\chi_{m} H^{2}\right),
$$


5.3 .

donde

$$
\vec{B}=\mu_{0}(\vec{H}+\vec{M}) .
$$

Como ya hemos discutido anteriormente, si consideramos un medio que cumpla con la siguiente relación, análoga a la ecuación de Clausius - Mossotti en el caso electrostático

$$
\chi_{m}=\rho_{m} \frac{\partial \mu_{r}}{\partial \rho_{m}},
$$

obtenemos que

$$
\nabla \cdot\left[\mathbf{T}_{M}+\frac{1}{2} \mathbf{I}(\vec{M} \cdot \vec{H})\right]=\vec{j} \times \vec{B}-\frac{1}{2} \mu_{0} H^{2} \nabla \mu_{r}+\frac{1}{2} \mu_{0} \nabla\left(\rho_{m} \frac{\partial \mu_{r}}{\partial \rho_{m}} H^{2}\right),
$$

donde el miembro derecho de esta ecuación es la densidad de fuerza magnética sobre un fluido, con esto hemos mostrado que la densidad de fuerza sobre un fluido, debida a campos magnetostáticos pueden obtenerse del miembro derecho de la ecuación de balance de momento (5.86). Considerando estos resultados obtenidos, ahora

es conveniente considerar el caso estático de la ecuación de balance de momento electromagnético (5.13), es decir

$$
\nabla \cdot \mathbf{T}_{E M}=\rho \vec{E}+\vec{j} \times \vec{B}+\frac{1}{2}[(\nabla \vec{E}) \cdot \vec{D}-(\nabla \vec{D}) \cdot \vec{E}+(\nabla \vec{H}) \cdot \vec{B}-(\nabla \vec{B}) \cdot \vec{H}]
$$

usando ecuaciones constitutivas en el miembro derecho de esta ecuación, hemos pues, obtenido resultados congruentes con los encontrados por Campos et al en [16], con esto podemos escribir un caso particular de la ecuación de balance (5.95) como

$$
\begin{aligned}
\nabla \cdot & {\left[\vec{D} \vec{E}+\vec{B} \vec{H}+\frac{1}{2} \mathbf{I}\left(\epsilon_{0} E^{2}+\mu_{0} H^{2}\right)\right]=\rho \vec{E}+\vec{j} \times \vec{B} } \\
& +\frac{1}{2} \epsilon_{0}\left[\nabla\left(\rho_{m} \frac{\partial \epsilon_{r}}{\partial \rho_{m}} E^{2}\right)-E^{2} \nabla \epsilon_{r}\right]+\frac{1}{2} \mu_{0}\left[\nabla\left(\rho_{m} \frac{\partial \mu_{r}}{\partial \rho_{m}} H^{2}\right)-H^{2} \nabla \mu_{r}\right] .
\end{aligned}
$$

Esta ecuación muestra como la ecuación de balance de momento (5.13) bajo ciertas consideraciones puede escribirse como (5.96), donde el miembro derecho de esta ecuación contiene las densidades de fuerzas propuestas por autores como Landau- Lifshitz [10] y Robinson [6]

\subsubsection{Densidad de fuerza magnética en sólidos}

Para deducir la densidad de fuerza en un sólido elástico, a partir de nuestra ecuación (5.86), escribimos dicha ecuación en componentes

$$
\frac{\partial}{\partial r_{j}}\left[B_{i} H_{j}-\frac{1}{2}\left(B_{l} H_{l}\right) \delta_{i j}\right]=(\vec{j} \times \vec{B})_{i}-\frac{1}{2}\left[\left(\frac{\partial B_{j}}{\partial r_{i}}\right) H_{j}-\left(\frac{\partial H_{j}}{\partial r_{i}}\right) B_{j}\right] .
$$


por otra parte, consideramos la ecuación constitutiva (4.45), podemos escribir la ecuación (5.97) como

$$
\frac{\partial}{\partial r_{j}}\left[B_{i} H_{j}-\frac{1}{2}\left(B_{l} H_{l}\right) \delta_{i j}\right]=(\vec{j} \times \vec{B})_{i}-\frac{1}{2} \mu_{0}\left[\left(\frac{\partial \mu_{j k} H_{k}}{\partial r_{i}}\right) H_{j}-\left(\frac{\partial H_{j}}{\partial r_{i}}\right) \mu_{j k} H_{k}\right] .
$$

Desarrollando de manera análoga al caso electrostático resulta

$$
\frac{\partial}{\partial r_{j}}\left[B_{i} H_{j}-\frac{1}{2}\left(B_{l} H_{l}\right) \delta_{i j}\right]=(\vec{j} \times \vec{B})_{i}-\frac{1}{2} \mu_{0} H_{k} H_{j}\left(\frac{\partial \mu_{j k}}{\partial r_{i}}\right) .
$$

Esta es la forma que toma nuestra ecuación de balance de momento, cuando consideramos un sólido en presencia de un campo magnetostático. Ahora análogamente a como lo hicimos para la densidad de fuerza sobre un fluido magnético, mostramos que esta ecuación (5.99) contiene a la densidad de fuerza sobre un sólido magnético. Para mostrarlo consideramos que la relación constitutiva puede expresarse en este caso de la siguiente forma

$$
B_{l}=\mu_{0}\left(\delta_{k l}+\chi_{(m) l k}\right) H_{k},
$$

si sustituimos esta ecuación en el miembro izquierdo de la ecuación (5.99) tenemos

$$
\frac{\partial}{\partial r_{j}}\left[B_{i} H_{j}-\frac{1}{2} \mu_{0}\left(\delta_{k l}+\chi_{(m) l k}\right) H_{k} H_{l} \delta_{i j}\right]=(\vec{J} \times \vec{B})_{i}-\frac{1}{2} \mu_{0} H_{k} H_{j}\left(\frac{\partial \mu_{j k}}{\partial r_{i}}\right),
$$

realizando los productos, reescribimos esta ecuación como

$$
\frac{\partial}{\partial r_{j}}\left(B_{i} H_{j}-\frac{1}{2} \epsilon_{0} H^{2} \delta_{i j}\right)=(\vec{j} \times \vec{B})_{i}-\frac{1}{2} \mu_{0} H_{k} H_{j}\left(\frac{\partial \mu_{j k}}{\partial r_{i}}\right)+\frac{\partial}{\partial r_{j}}\left(\frac{1}{2} \mu_{0} H_{k} H_{l} \chi_{(m) l k} \delta_{i j}\right) .
$$

Ahora, de una forma equivalente a como lo hicimos en el caso electrostático, analizamos el término $\chi_{(m) l k} \delta_{i j}$, el cual es un tensor de cuarto rango, simétrico en los índices $i$ y $j$ debido a las propiedades de $\delta$, y simétrico en los índices $l$ y $k$. Entonces de manera similar con el caso electrostático definimos

$$
\chi_{(m) l k} \delta_{i j} \equiv-b_{l k i j},
$$

donde $b_{l k i j}$ es un tensor de cuarto rango, equivalente al propuesto por Stratton [1]. Escribiendo la ecuación (5.102) en términos del tensor $b_{l k i j}$, tenemos

$$
\frac{\partial}{\partial r_{j}}\left(B_{i} H_{j}-\frac{1}{2} \mu_{0} H_{l} H_{l} \delta_{i j}\right)=(\vec{j} \times \vec{B})_{i}-\frac{1}{2} \mu_{0} H_{k} H_{j}\left(\frac{\partial \mu_{j k}}{\partial r_{i}}\right)+\frac{\partial}{\partial r_{j}}\left(-\frac{1}{2} \mu_{0} H_{k} H_{l} b_{l k i j}\right),
$$

en esta ecuación podemos identificar a la densidad de fuerza como

$$
f_{(m) i}=(\vec{j} \times \vec{B})_{i}-\frac{1}{2} \mu_{0} H_{k} H_{j}\left(\frac{\partial \mu_{j k}}{\partial r_{i}}\right)+\frac{\partial}{\partial r_{j}}\left(-\frac{1}{2} \mu_{0} H_{k} H_{l} b_{l k i j}\right) .
$$


Esta densidad de fuerza $f_{(m) i}$, es equivalente a la densidad de fuerza de la ecuación (4.81) encontrada por Stratton [1] (pag. 154), Robinson [4] y Landau- Lifshitz [10] entre otros. Con esto mostramos una nueva forma para calcular las densidades de fuerza sobre fluidos y sólidos, debidas a campos electrostáticos y magnetostáticos, lo cual es una muestra del alcance de la ecuación de balance de momento electromagnético (5.13) obtenida a partir de las ecuaciones de Maxwell. Estos son elementos que apoyan el hecho de considerar que la ecuación de balance de momento electromagnético (5.13) ya contiene las fuerzas necesarias para analizar al menos a los fluidos y sólidos en presencia de campos estáticos.

A demás, este método de calcular las densidades de fuerza a partir de la ecuación de balance (5.13), nos permite entre otras cosas, apreciar la simetría del intercambio entre los vectores $\vec{E}$ y $\vec{H}$.

\subsection{La densidad de fuerza en medios materiales}

En el capítulo anterior hemos mostrado los resultados obtenidos por Robinson [4], Stratton [1], Panofsky y Phillips [2], Tamm [9], Landau y Lifshitz [10] para las densidades de fuerza en fluidos y sólidos, distinguiendo si estas densidades de fuerza son debidas a campos electrostáticos o magnetostáticos, ademas de mostrar la relación que existe entre estas densidades de fuerza. Por otra parte, en este capítulo demostramos que estas densidades de fuerza pueden obtenerse directamente de la ecuación de balance de momento electromagnético y densidad de fuerza. Con estos antecedentes podemos preguntarnos, ¿que tan general es la densidad de fuerza obtenida directamente de la ecuación de balance?

En este sección damos mayor sustento a la propuesta hecha por CJL [16], de una expresión general para la densidad de fuerza en medios. Este sustento esta basado en los resultados mostrados y obtenidos en los capítulos previos, para ello partimos de la ecuación de balance de momento electromagnético para campos independientes del tiempo

$$
\nabla \cdot \mathbf{T}_{E M}=\rho \vec{E}+\vec{j} \times \vec{B}+\frac{1}{2}[(\nabla \vec{E}) \cdot \vec{D}-(\nabla \vec{D}) \cdot \vec{E}+(\nabla \vec{H}) \cdot \vec{B}-(\nabla \vec{B}) \cdot \vec{H}]
$$

donde podemos reconocer al miembro derecho de esta ecuación como una densidad de fuerza, la cual denotaremos como $\vec{f}_{G}$, es decir

$$
\vec{f}_{G}=\rho \vec{E}+\vec{j} \times \vec{B}+\frac{1}{2}[(\nabla \vec{E}) \cdot \vec{D}-(\nabla \vec{D}) \cdot \vec{E}+(\nabla \vec{H}) \cdot \vec{B}-(\nabla \vec{B}) \cdot \vec{H}]
$$

establecido esto, vamos a mostrar que $\vec{f}_{G}$ es la densidad de fuerza generalizada en medios.

Si consideramos un sistema en el cual sólo están presentes campos electrostáticos, la densidad de fuerza $\vec{f}_{G}$ toma la siguiente forma 


$$
\vec{f}_{G E}=\rho \vec{E}+\frac{1}{2}[(\nabla \vec{E}) \cdot \vec{D}-(\nabla \vec{D}) \cdot \vec{E}] .
$$

Ahora, puesto que en el capítulo anterior se mostró que la densidad de fuerza sobre un fluido es un caso particular de la densidad de fuerza en un sólido, vamos a analizar a la densidad de fuerza $\vec{f}_{G E}$ para un sólido. Dicho esto consideramos la ecuación constitutiva (5.43) y sustituyendo en la ecuación (5.108) obtenemos

$$
\begin{aligned}
f_{i(G E)} & =\rho E_{i}+\frac{1}{2}\left[\left(\frac{\partial}{\partial r_{i}} E_{j}\right) D_{j}-\left(\frac{\partial}{\partial r_{i}} D_{j}\right) E_{j}\right] \\
& =\rho E_{i}+\frac{1}{2}\left[\left(\frac{\partial}{\partial r_{i}} E_{j}\right) D_{j}-\left(\frac{\partial}{\partial r_{i}} \epsilon_{0} \epsilon_{j k} E_{k}\right) E_{j}\right] \\
& =\rho E_{i}+\frac{1}{2}\left\{\left(\frac{\partial}{\partial r_{i}} E_{j}\right) D_{j}-\epsilon_{0}\left[\left(\frac{\partial}{\partial r_{i}} \epsilon_{j k}\right) E_{k}+\epsilon_{j k}\left(\frac{\partial}{\partial r_{i}} E_{k}\right)\right] E_{j}\right\} \\
& =\rho E_{i}+\frac{1}{2}\left[D_{j}\left(\frac{\partial}{\partial r_{i}} E_{j}\right)-D_{k}\left(\frac{\partial}{\partial r_{i}} E_{k}\right)-\left(\frac{\partial}{\partial r_{i}} \epsilon_{j k}\right) \epsilon_{0} E_{k} E_{j}\right],
\end{aligned}
$$

analizando el término $D_{j}\left(\frac{\partial}{\partial r_{i}} E_{j}\right)-D_{k}\left(\frac{\partial}{\partial r_{i}} E_{k}\right)$, y considerando que los índices $j$ y $k$ son mudos, entonces si $j \rightarrow k$, el término se anula, puesto que

$$
D_{j}\left(\frac{\partial}{\partial r_{i}} E_{j}\right)-D_{k}\left(\frac{\partial}{\partial r_{i}} E_{k}\right)=0
$$

con esta consideración, la ecuación (5.108) se escribe como

$$
f_{i(G E)}=\rho E_{i}-\frac{1}{2} \epsilon_{0} E_{k} E_{j}\left(\frac{\partial}{\partial r_{i}} \epsilon_{j k}\right) .
$$

Siguiendo el razonamiento de la sección (4.2.2), es decir, redefiniendo el gradiente

$$
\nabla_{i} \rightarrow\left(\frac{\partial}{\partial r_{i}}+\frac{\partial S_{l m}}{\partial r_{i}} \frac{\partial}{\partial S_{l m}}\right)
$$

y usando esta definición en la ecuación (5.110), obtenemos que

$$
\begin{aligned}
f_{i(G E)} & =\rho E_{i}-\frac{1}{2} \epsilon_{0} E_{k} E_{j}\left(\frac{\partial}{\partial r_{i}}+\frac{\partial S_{l m}}{\partial r_{i}} \frac{\partial}{\partial S_{l m}}\right)\left(\epsilon_{j k}\right) \\
& =\rho E_{i}-\frac{1}{2} \epsilon_{0} E_{k} E_{j}\left(\frac{\partial \epsilon_{j k}}{\partial r_{i}}\right)-\frac{1}{2} \epsilon_{0} E_{k} E_{j} \frac{\partial S_{l m}}{\partial r_{i}}\left(\frac{\partial \epsilon_{j k}}{\partial S_{l m}}\right) .
\end{aligned}
$$

Claramente esta densidad de fuerza es igual al miembro derecho de ecuación (5.66), la cual analizamos y demostramos que conduce a la densidad de fuerza en sólidos usual. Con esto es evidente que la densidad de fuerza $f_{i(G E)}$ en un caso particular se convierte 


\section{4 .}

en la densidad de fuerza en un sólido $f_{i(s)}$, y a su vez la densidad de fuerza de $f_{i(s)}$ en un caso particular se convierte en la densidad de fuerza de Helmholtz $f_{i(H)}$, es decir

$$
f_{i(G)} \rightarrow f_{i(G E)} \rightarrow f_{i(s)} \rightarrow f_{i(H)}
$$

Cuando consideramos unicamente campos magnetostáticos, la densidad de fuerza $\overrightarrow{f_{G}}$ toma la forma de

$$
\vec{f}_{G M}=\vec{j} \times \vec{B}+\frac{1}{2}[(\nabla \vec{H}) \cdot \vec{B}-(\nabla \vec{B}) \cdot \vec{H}],
$$

análogamente como lo hicimos para el caso electrostático se puede encontrar que la densidad de fuerza $f_{G M}$ ) contiene las densidades de fuerza usualmente conocidas, con esto podemos ver que

$$
f_{i(G)} \rightarrow f_{i(G M)} \rightarrow f_{i(s M)} \rightarrow f_{i(H M)}
$$

Con esto hemos mostrado como la densidad de fuerza $f_{i(G)}$ la cual se obtuvo directamente de la ecuación de balance de momento electromagnético (5.106), contiene, como casos particulares, las densidades de fuerza deducidas y discutidas en los capítulos anteriores, con esto sustentamos la propuesta de la densidad de fuerza (5.107), como la densidad de fuerza en medios materiales. 


\section{Capítulo 6}

\section{Equivalencia de las diferentes propuestas para el tensor de esfuerzos en un sólido elástico}

En este capitulo buscamos mostrar la equivalencia de las propuestas de los autores Stratton, Landau-Lifshitz y Robinson para el tensor de esfuerzos en un sólido elástico.

Stratton [1] escribe el tensor como

$$
t_{i j}=\epsilon_{0}\left(\epsilon+\frac{a_{2}-a_{1}}{2}\right) E_{i} E_{j}-\epsilon_{0}\left(\frac{\epsilon+a_{2}}{2}\right) E^{2} \delta_{i j},
$$

Landau y Lifshitz [10] por su parte escriben el tensor como

$$
\sigma_{i k}=\epsilon_{0}\left(\epsilon-\frac{a_{1}}{2}\right) E_{i} E_{k}-\epsilon_{0}\left(\frac{\epsilon+a_{2}}{2}\right) E^{2} \delta_{i k}
$$

y Robinson [4], escribe

$$
T_{i j}^{e}+T_{i j}^{e s}=\frac{1}{2}\left(E_{i} D_{j}+E_{j} D_{i}-\vec{E} \cdot \vec{D} \delta_{i j}\right)-\frac{1}{2} \epsilon_{0} \alpha_{k l i j} E_{k} E_{l},
$$

la cual es una expresión que no es directamente comparable con los tensores escritos por Stratton y Landau- Lifshitz, esto es debido a que el tensor (6.3) está expresado en términos del tensor $\alpha_{k l i j}$.

Para darnos cuenta si son o no equivalente estas propuestas es necesario unificar las definiciones de las constantes utilizadas por dichos autores. Estas constantes surgen tras analizar el tensor

$$
\alpha_{j k l m}=\frac{\partial \epsilon_{j k}}{\partial S_{l m}}
$$

Estos autores señalan que el tensor $\alpha_{j k l m}$ es un tensor de constantes y que en el caso de un sólido elástico existen únicamente 2 constantes independientes, pero, sólo Stratton muestra con detalle como se obtienen estas dos constantes, la confusión surge 
cuando estas constantes aparecen en el tensor electrostático asociado al estudio de la densidad de fuerza en sólidos ya que los tres autores usan las mismas letras para estas constantes, pero no es evidente que representen las mismas cosas.

\subsection{Equivalencia de las propuestas para el tensor de esfuerzos de Landau- Lifshitz y Robinson}

En el estudio de la densidad de fuerza en un sólido, Robinson comenta que sólo existen dos constantes independientes y escribe que para este caso en particular el tensor $T_{i j}^{e s}$ debe de ser

$$
T_{i j}^{e s}=-\frac{1}{2} \epsilon_{0}\left(a_{1} E_{i} E_{j}+a_{2} E^{2} \delta_{i j}\right),
$$

donde $a_{1}$ y $a_{2}$ son dichas constantes, además si consideramos que

$$
E_{i} D_{j}=E_{j} D_{i}
$$

y

$$
D_{j}=\epsilon_{0} \epsilon E_{j},
$$

podemos escribir una expresión para el tensor propuesto por Robinson, la cual puede ser comparable con las expresiones escritas por Landau-Lifshitz y Stratton. Entonces aplicando las ecuaciones $(6.5),(6.6)$ y (6.7) en la ecuación (6.3), obtenemos

$$
T_{i j}^{e}+T_{i j}^{e s}=\epsilon_{0}\left[\left(\epsilon-\frac{a_{1}}{2}\right) E_{i} E_{j}-\left(\frac{\epsilon+a_{2}}{2}\right) E^{2} \delta_{i k}\right] .
$$

Ahora ya podemos hacer una comparación inmediata entre los tensores (6.2) y (6.8). Es claro que los tensores escritos por Landau-Lifshitz y Robinson son equivalentes, es decir

$$
\sigma_{i k} \equiv T_{i j}^{e}+T_{i j}^{e s}
$$

puesto que

$$
\sigma_{i k}=\epsilon_{0}\left(\epsilon-\frac{a_{1}}{2}\right) E_{i} E_{k}-\epsilon_{0}\left(\frac{\epsilon+a_{2}}{2}\right) E^{2} \delta_{i k}
$$

y

$$
T_{i j}^{e}+T_{i j}^{e s}=\epsilon_{0}\left[\left(\epsilon-\frac{a_{1}}{2}\right) E_{i} E_{j}-\left(\frac{\epsilon+a_{2}}{2}\right) E^{2} \delta_{i k}\right] .
$$

Debido a que Robinson no escribió una expresión como la ecuación (6.8), la comparación entre sus resultados y los obtenidos por Landau y Lifshitz no es obvia.

\subsection{Equivalencia de las propuestas para el tensor de esfuerzos de Stratton y Landau- Lifshitz}

Ahora falta realizar la comparación con el tensor escrito por Stratton. Para facilitar esta comparación, escribimos nuevamente el tensor de Stratton pero cambiando las 
constantes $a_{1}=\alpha_{1}$ y $a_{2}=\alpha_{2}$ junto con el tensor escrito por Landau y Lifshitz, con el fin de distinguir las constantes, entonces tenemos

$$
t_{i j}=\epsilon_{0}\left(\epsilon+\frac{\alpha_{2}-\alpha_{1}}{2}\right) E_{i} E_{j}-\epsilon_{0}\left(\frac{\epsilon+\alpha_{2}}{2}\right) E^{2} \delta_{i j},
$$

y

$$
\sigma_{i k}=\epsilon_{0}\left(\epsilon-\frac{a_{1}}{2}\right) E_{i} E_{k}-\epsilon_{0}\left(\frac{\epsilon+a_{2}}{2}\right) E^{2} \delta_{i k} .
$$

Ahora nuestro objetivo es aclarar como se relacionan las constantes de cada autor, para establecer si los tensores (6.9) y (6.10) son equivalentes. Para esto, vamos a utilizar el hecho de que Stratton obtiene las siguientes relaciones para sus constantes

$$
\alpha_{j j j j}=\alpha_{1}, \quad \alpha_{j j k k}=\alpha_{2}, \quad \alpha_{j k j k}=\alpha_{3}, \quad(j \neq k) .
$$

y

$$
\alpha_{1}-\alpha_{2}-4 \alpha_{3}=0, \quad \alpha_{3}=\frac{1}{4}\left(\alpha_{1}-\alpha_{2}\right) .
$$

Con ayuda de estas ecuaciones, muestra como el tensor de cuarto rango $\alpha_{j k l m}$, para un sólido, tiene unicamente dos constantes independientes, denominadas por Stratton como, $\alpha_{1}$ y $\alpha_{2}$. Aquí surge el punto clave, Stratton escribe el tensor en términos de las constantes $\alpha_{1}$ y $\alpha_{2}$, pero esta no es la única forma de escribirlo, si elegimos expresar este tensor (6.9) en función de las constantes $\alpha_{3}$ y $\alpha_{2}$ y dado que

$$
-2 \alpha_{3}=\frac{1}{2}\left(\alpha_{1}-\alpha_{2}\right),
$$

obtenemos

$$
t_{i j}=\epsilon_{0}\left(\epsilon-2 \alpha_{3}\right) E_{i} E_{j}-\epsilon_{0}\left(\frac{\epsilon+\alpha_{2}}{2}\right) E^{2} \delta_{i j} .
$$

Ahora, una vez escrito el tensor de Stratton de la forma (6.14), hacemos una comparación entre los tensores (6.8), (6.10) y (6.14), es decir

$$
\begin{gathered}
\sigma_{i k}=\epsilon_{0}\left(\epsilon-\frac{a_{1}}{2}\right) E_{i} E_{k}-\epsilon_{0}\left(\frac{\epsilon+a_{2}}{2}\right) E^{2} \delta_{i k}, \\
T_{i j}^{e}+T_{i j}^{e s}=\epsilon_{0}\left[\left(\epsilon-\frac{a_{1}}{2}\right) E_{i} E_{j}-\left(\frac{\epsilon+a_{2}}{2}\right) E^{2} \delta_{i k}\right],
\end{gathered}
$$

y

$$
t_{i j}=\epsilon_{0}\left(\epsilon-2 \alpha_{3}\right) E_{i} E_{j}-\epsilon_{0}\left(\frac{\epsilon+\alpha_{2}}{2}\right) E^{2} \delta_{i j} .
$$

Con esta comparación, podemos ver, que los tensores de Stratton, Landau y Robinson, son equivalentes, además podemos establecer una igualdad entre las constantes de Landau y Robinson con las constantes de Stratton, la cual queda expresada en las siguientes ecuaciones

$$
4 \alpha_{3}=a_{1}
$$

y

$$
\alpha_{2}=a_{2} .
$$


Con esto establecemos que los tensores (6.8), (6.10) y (6.14) son iguales y hemos unificado el lenguaje de estos tres autores para así facilitar el análisis siguiente.

\subsection{Demostración de que en el tensor de Maxwell es- tán incluidas las propuestas de Stratton, Landau- Lifshitz y Robinson}

En esta sección buscamos mostrar, si es posible o no, obtener el tensor que proponen Stratton, Landau-Lifshitz y Robinson para un sólido elástico sometido a un campo electrostático, directamente del tensor de Maxwell en medios. Para esto partimos de la expresión bien conocida de dicho tensor

$$
T_{i j}=D_{i} E_{j}-\frac{1}{2} D_{k} E_{k} \delta_{i j}
$$

ahora, es necesario escribir la ecuación constitutiva que vincula los campos $\vec{D}$ y $\vec{E}$

$$
D_{i}=\epsilon_{0} \epsilon_{i l} E_{l},
$$

es aquí precisamente donde radica el meollo de este problema, puesto que nosotros hacemos énfasis en que dicha ecuación constitutiva debe contener las características del sistema al que deseemos tratar, consideramos que la permitividad $\epsilon_{i l}$ escrita en la ecuación constitutiva es el vehículo en el cual se deben de contener las características del sistema.

Landau y Lifshitz [10], en el caso de un sólido expresa que la permitividad está compuesta por dos partes, una parte $\epsilon_{i l}^{0}$, que representa las propiedades del medio antes de ser deformado y otra $a_{i l m n} S_{m n}$ que contiene la información del medio deformado, es decir

$$
\epsilon_{i l}=\epsilon_{i l}^{0}+a_{i l m n} S_{m n} .
$$

Para comprender mejor estas propiedades de la permitividad $\epsilon_{i l}$, recordemos que en el cálculo de Stratton, ya se sigue esta linea de razonamiento, pero nunca escribe explícitamente una expresión como (6.19) y utiliza un lenguaje sumamente, anticuado, pone cuidado en considerar la permitividad del medio antes y después de la deformación. En lo que respecta a Robinson el lenguaje es mas claro y moderno, pero no tiene cuidado en hacer distinción de las partes que constituyen la permitividad, puesto que abusa de la letra $\epsilon$, lo cual puede dar pie a muchas confusiones.

Una vez expuestas estas consideraciones proponemos el tensor permitividad como la suma de dos tensores, $\epsilon_{i l}^{0}$ y $\epsilon_{i l}^{*}$, donde $\epsilon_{i l}^{0}$ representa, al igual que para Landau y Lifshitz, la permitividad del medio antes de ser deformado y $\epsilon_{i l}^{*}$ contiene las propiedades del medio deformado, de tal forma que

$$
\epsilon_{i l}=\epsilon_{i l}^{0}+\epsilon_{i l}^{*},
$$


Si sustituimos las ecuaciones (5.43) y (6.20) en la expresión para el tensor de Maxwell (6.17), obtenemos

$$
T_{i j}=\left(\epsilon_{i l}^{0}+\epsilon_{i l}^{*}\right) E_{l} E_{j}-\frac{1}{2}\left(\epsilon_{m k}^{0}+\epsilon_{m k}^{*}\right) E_{m} E_{k} \delta_{i j},
$$

ahora, definimos

$$
\begin{gathered}
\epsilon_{i l}^{0} E_{l}=\epsilon E_{i}, \\
\epsilon_{i l}^{*} E_{l}=\alpha E_{i}, \\
\epsilon_{m k}^{0} E_{m}=\epsilon E_{k},
\end{gathered}
$$

y

$$
\epsilon_{m k}^{*} E_{m}=\beta E_{k},
$$

donde $\alpha$ y $\beta$ son dos constantes. Con esto podemos escribir la ecuación (6.21) como

$$
T_{i j}=\epsilon_{0}(\epsilon+\alpha) E_{i} E_{j}-\frac{1}{2} \epsilon_{0}(\epsilon+\beta) E^{2} \delta_{i j},
$$

si comparamos con el tensor de Stratton

$$
t_{i j}=\epsilon_{0}\left(\epsilon+\frac{a_{2}-a_{1}}{2}\right) E_{i} E_{j}-\epsilon_{0}\left(\frac{\epsilon+a_{2}}{2}\right) E^{2} \delta_{i j},
$$

basta con definir las constantes

$$
\alpha=\frac{a_{2}-a_{1}}{2}
$$

y

$$
\beta=a_{2} .
$$

Con esto mostramos que el tensor de Maxwell en medios materiales, bajo ciertas consideraciones, reproduce el tensor propuesto por Stratton, Landau-Lifshitz y Robinson para medios sólidos. Esto es un punto muy importante para nuestro trabajo, puesto que es un elemento más que apoya nuestra visión, las ecuaciones de Maxwell más ecuaciones constitutivas y el cálculo vectorial y tensorial proveen los tensores y las densidades de fuerza en medios materiales. 
6.3. 


\section{Capítulo 7}

\section{Conclusiones}

En este trabajo hemos mostrado algunas de las deducciones usuales de las densidades de fuerza en medios materiales, hemos seguido el desarrollo de estas deducciones señalando los puntos delicados de las mismas y hemos establecido las equivalencias que existen entre estas deducciones al unificar el lenguaje de las mismas. Además no solo nos limitamos a analizar las deducciones usuales, por otra parte también demostramos que la densidad de fuerza sobre un sólido debida a campos electrostáticos o magnetostáticos puede obtenerse directamente de la ecuación de balance de momento electromagnético deducida de las ecuaciones de Maxwell; previamente Campos et al [15] demostraron que efectivamente esta ecuación de balance provee la densidad de fuerza sobre un fluido, es decir la densidad de fuerza de Helmholtz.

En este trabajo mostramos la generalidad de la ecuación de balance de momento que surge de las ecuaciones de Maxwell, con las relaciones constitutivas adecuadas es posible deducir de ella las densidades de fuerza en fluidos y en sólidos ya conocidas, se tiene así una densidad de fuerza en general para medios materiales por lo cual no hace falta buscar dichas densidades en otro lado, este es un método novedoso, sustentado en una interpretación diferente de la ecuación de balance de momento, puesto que, usualmente se deduce primero una densidad de fuerza a partir de la energía libre del sistema [Stratton, Tamm, Panofsky y Phillips, Landau y Lifshitz], y posteriormente se realiza el balance de momento, hemos demostrado que esto es innecesario. Este es método puramente macroscópico, a diferencia de lo hecho por Gordon [5], Peierls [8], Lai et al en [11] y [12] entre otros.

Con esto mostramos la enorme riqueza de la teoría de Maxwell, las cuatro ecuaciones y las relaciones constitutivas conducen a las densidades de fuerza en medios materiales conocidas. 
7.0 . 


\section{Bibliografía}

[1] Stratton J. A. (1941), Electromagnetic Theory, Mc Graw-Hill, New York and London

[2] Panofsky W. K. H. y Phillips M. (1962), Classical Electricity and Magnetism, 2nd Addison-Wesley, Mass.

[3] Landau L. D. and Lifshitz E. M. (1969), Teoría de la elasticidad, Reverté, Barcelona.

[4] Robinson F. N. H. (1973), Macroscopic Electromagnetism, Pergamon, New York.

[5] Gordon, J. (1973) Phys. Rev. A 8, 14.

[6] Robinson, F. N. H. (1975) Phys. Rep. 16C, 314.

[7] Jackson J. D. (1975), Classical Electrodynamics, 2nd Wiley, New York.

[8] Peierls R. (1976)Proc. R. Soc. Lond. A 374, 475.

[9] Tamm I. Y. (1979), Fundamentos de la teoría de la electricidad, Mir, Moscú

[10] Landau L. D. and Lifshitz E. M. (1981), Electrodinámica de los medios continuos, Reverté, Barcelona.

[11] Lai H. M., Suen W. M., and Young K. (1982) Phys. Rev. A 25, 3.

[12] Lai H. M., C. K. Ng, and Young K. (1984) Phys. Rev. A 30, 2. 
[13] Peierls R. (1991) More Surprises in Theoretical Physics., New Jersey: Princeton.

[14] W. Michael Lai, David Rubin and Erhard Krempl (1996) Introduction to Continuum Mechanics, Butterworth-Heinemann, Reino Unido.

[15] Campos I. , Jiménez J.L. and López Mariño M.A. (2010), Nuovo Cimento, 125 B, N.4.

[16] Campos I. , Jiménez J.L. and López Mariño M.A. (2012), Revista Brasileira de Ensino de Fisica, 2, 2303. 
Apéndices 



\section{Apéndice A}

\section{Elasticidad}

\section{A.1. Descripción material y descripción espacial}

Cuando un medio continuo está en movimiento, las cantidades tensoriales que se asocian con las partículas especificas (temperatura $\theta$, velocidad $\vec{v}$, etc) cambian con el tiempo. Podemos describir estos cambios por:

1 siguiendo las partículas, es decir, expresamos $\theta, \vec{v}$ como función de las partículas (denotado por las coordenadas materiales $X_{i}$ ) y el tiempo. En otras palabras, expresamos

$$
\begin{aligned}
& \theta=\theta\left(X_{i}, t\right), \\
& \vec{v}=\vec{v}\left(X_{i}, t\right) .
\end{aligned}
$$

Tal descripción se conoce como la descripción material. Otros nombres para la misma son: Descripción lagrangiana y descripción de referencia.

2 Observando los cambios de las posiciones fijas, es decir, expresamos $\theta, \vec{v}$ como función de las posiciones fijas y el tiempo, es decir

$$
\begin{aligned}
& \theta=\theta\left(x_{i}, t\right), \\
& \vec{v}=\vec{v}\left(x_{i}, t\right) .
\end{aligned}
$$

Tal descripción se conoce como una descripción espacial o descripción euleriana. $x_{i}$ localiza la posición fija de puntos en el espacio físico y es conocido como el espacio de coordenadas. Las coordenadas espaciales $x_{i}$ de una partícula en cualquier tiempo $t$ están relacionados con las coordenadas materiales $X_{i}$ de la partícula por la siguiente ecuación

$$
x_{i}=x_{i}\left(X_{i}, t\right)
$$

\section{A.2. Derivada material}

La tasa de cambio en el tiempo de una cantidad (tal como la temperatura o la velocidad o tensor de tensiones) de una partícula de material, se conoce como una derivada de material. Vamos a denotar la derivada material por $D / D t$. 
(I) Cuando se utiliza una descripción material de la cantidad, tenemos

$$
\theta=\theta\left(X_{i}, t\right)
$$

entonces

$$
\frac{D \theta}{D t}=\left.\frac{\partial \theta}{\partial t}\right|_{X_{i} f i j o}
$$

(II) Cuando se utiliza una descripción espacial de la cantidad, tenemos

$$
\theta=\theta\left(x_{i}, t\right)
$$

entonces

$$
\frac{D \theta}{D t}=\frac{\partial \theta}{\partial x_{i}} \frac{\partial x_{i}}{\partial t}+\left(\frac{\partial \theta}{\partial t}\right)_{x_{i} f i j o}
$$

Cuando se utilizan coordenadas cartesianas rectangulares, $\frac{\partial x_{i}}{\partial t}$ son las componentes de la velocidad $v_{i}$ de la partícula. De este modo, la derivada material en coordenadas rectangulares es

$$
\frac{D \theta}{D t}=\frac{\partial \theta}{\partial t}+\vec{v} \cdot \nabla \theta
$$

donde hay que destacar que estas ecuaciones son para $\theta$ en una descripción espacial, es decir, $\theta=\theta\left(x_{i}, t\right)$.

\section{A.3. Tensor de deformación}

Hay muchos problemas para los cuales el desplazamiento de cada punto material es muy pequeña. En esta sección, se deriva el tensor de la cual que caracteriza a la deformación.

Considerando un cuerpo, que tiene una configuración particular, en algún tiempo de referencia $t_{0}$, los cambios en otra configuración en el instante t. Haciendo referencia a la Fig. 3,3, un punto material $\mathrm{P}$ típico experimenta un desplazamiento $\vec{u}$, de modo que llega a la posición

$$
\vec{x}=\vec{X}+\vec{u}(\vec{X}, t)
$$

Un punto vecino $\mathrm{Q} \vec{X}+d \vec{X}$ llega a $\vec{x}+d \vec{x}$ que está relacionado con $\vec{X}+d \vec{X}$ por:

$$
\vec{x}+d \vec{x}=\vec{X}+d \vec{X}+\vec{u}(\vec{X}+d \vec{X}, t)
$$

Restando las ecuaciones anteriores se obtiene

$$
d \vec{x}=d \vec{X}+\vec{u}(\vec{X}+d \vec{X}, t)-\vec{u}(\vec{X}, t)
$$

Utilizando la definición de gradiente de una función vectorial tenemos

$$
d \vec{x}=d \vec{X}+(\nabla \vec{u}) d \vec{X}
$$


donde $\nabla \vec{u}$ es un tensor de segundo orden conocido como el gradiente de desplazamiento. Podemos escribir las ecuación anterior como

$$
d \vec{x}=(\mathbf{I}+\nabla \vec{u}) \vec{X}
$$

definiendo

$$
\mathbf{A}=(\mathbf{I}+\nabla \vec{u})
$$

tenemos

$$
\begin{gathered}
d \vec{x}=\mathbf{A} d \vec{X} \\
d \vec{x} \cdot d \vec{x}=\mathbf{A} d \vec{X} \cdot \mathbf{A} d \vec{X}=d \vec{X} \cdot \mathbf{A}^{T} \mathbf{A} d \vec{X}
\end{gathered}
$$

es decir,

$$
(d s)^{2}=d \vec{X} \cdot \mathbf{A}^{T} \mathbf{A} d \vec{X}
$$

Si $\mathbf{A}$ es un tensor ortogonal, entonces $\mathbf{A}^{T} \mathbf{A}=\mathbf{I}, \mathbf{y}$

$$
(d s)^{2}=(d S)^{2}
$$

Por lo tanto, un tensor A ortogonal corresponde a un movimiento rígido del cuerpo (traslacióny / o rotación).

$$
\mathbf{A}^{T} \mathbf{A}=(\mathbf{I}+\nabla \vec{u})^{T}(\mathbf{I}+\nabla \vec{u})=\mathbf{I}+\nabla \vec{u}+(\nabla \vec{u})^{T}+(\nabla \vec{u})^{T}(\nabla \vec{u})
$$

si consideramos sólo los casos en que los componentes del vector de desplazamiento así como sus derivadas parciales son muy pequeños, de modo que el valor absoluto de cada componente de $(\nabla \vec{u})^{T}(\nabla \vec{u})$ es una cantidad más pequeña que las componentes de $\nabla \vec{u}$

Para tal caso, la ecuación anterior se convierte en:

$$
\mathbf{A}^{T} \mathbf{A} \approx \mathbf{I}+\nabla \vec{u}+(\nabla \vec{u})^{T} \equiv \mathbf{I}+2 \mathbf{S}
$$

donde

$$
\mathbf{S}=\frac{1}{2}\left[(\nabla \vec{u})^{T}+(\nabla \vec{u})\right]
$$

escribiendo en notación indicial

$$
S_{i j}=\frac{1}{2}\left(\frac{\partial u_{i}}{\partial r_{j}}+\frac{\partial u_{j}}{\partial r_{i}}\right),
$$

El tensor $\mathbf{S}$ caracteriza a los cambios de longitudes en el continuo de someterse a pequeñas deformaciones. Este tensor $\mathbf{S}$ se conoce como el tensor de deformaciones infinitesimales. 
A.3.

Tensor de deformación 


\section{Apéndice B}

\section{Deducción de la ecuación (4.33)}

Si $\theta$ es una cantidad escalar asociada con la materia moviéndose en un medio con una velocidad $\vec{v}$, la derivada total con respecto al tiempo está dada por

$$
\frac{d \theta}{d t}=\frac{\partial \theta}{\partial t}+\left(\frac{d u_{i}}{d t}\right) \frac{\partial \theta}{\partial r_{i}}
$$

donde la velocidad está definida como

$$
\frac{d u_{i}}{d t}=v_{i}
$$

Utilizando las ecuaciones (B.1) y (B.2) escribe una ecuación que relaciona las derivadas con respecto al tiempo totales y parciales de una cantidad que se mueve con una velocidad $v_{i}$, es decir

$$
\frac{\partial \theta}{\partial t}=\frac{d \theta}{d t}-v_{i} \frac{\partial \theta}{\partial r_{i}} .
$$

Por otra parte escribe el tensor $\frac{\partial u_{i}}{\partial r_{j}}$ en términos de su parte simétrica $S_{i j}$ y antisimetrica $W_{i j}$

$$
\frac{\partial u_{i}}{\partial r_{j}}=S_{i j}-W_{i j}
$$

donde

$$
S_{i j}=\frac{1}{2}\left(\frac{\partial u_{i}}{\partial r_{j}}+\frac{\partial u_{j}}{\partial r_{i}}\right)
$$

$\mathrm{y}$

$$
W_{i j}=\frac{1}{2}\left(\frac{\partial u_{j}}{\partial r_{i}}-\frac{\partial u_{i}}{\partial r_{j}}\right) .
$$

Derivando las ecuaciones (B.5) y (B.6)

$$
\begin{aligned}
\frac{d S_{i j}}{d t} & =\frac{1}{2}\left(\frac{\partial}{\partial r_{j}} \frac{d u_{i}}{d t}+\frac{\partial}{\partial r_{j}} \frac{d u_{j}}{\partial d t}\right) \\
& =\frac{1}{2}\left(\frac{\partial v_{i}}{\partial r_{j}}+\frac{\partial v_{j}}{\partial r_{i}}\right)
\end{aligned}
$$


B.0.

$$
\begin{aligned}
\frac{d W_{i j}}{d t} & =\frac{1}{2}\left(\frac{\partial}{\partial r_{i}} \frac{d u_{j}}{d t}-\frac{\partial}{\partial r_{j}} \frac{d u_{i}}{\partial d t}\right) \\
& =\frac{1}{2}\left(\frac{\partial v_{j}}{\partial r_{i}}-\frac{\partial v_{i}}{\partial r_{j}}\right)
\end{aligned}
$$

Considerando que la permitividad tiene la siguiente dependencia

$$
\epsilon_{i j} \equiv \epsilon_{i j}\left(S_{i j}, W_{i j}\right)
$$

escribe una derivada con respecto al tiempo de la forma

$$
\frac{d \epsilon_{i j}}{d t}=\epsilon_{i k}\left(\frac{d W_{j k}}{d t}\right)+\epsilon_{k j}\left(\frac{d W_{i k}}{d t}\right)+\left(\frac{d \epsilon_{i j}}{d S_{k l}}\right) \frac{d S_{k l}}{d t},
$$

esta es una expresión que no discute, la cual sirve como base de su cálculo, aparentemente es una especie de regla de la cadena. Sustituyendo las ecuaciones (B.7) y (B.8) en la expresión (B.10) obtiene

$$
\frac{d \epsilon_{i j}}{d t}=\epsilon_{i k} \frac{1}{2}\left(\frac{\partial v_{k}}{\partial r_{j}}-\frac{\partial v_{j}}{\partial r_{k}}\right)+\epsilon_{k j} \frac{1}{2}\left(\frac{\partial v_{k}}{\partial r_{i}}-\frac{\partial v_{i}}{\partial r_{k}}\right)+\alpha_{i j k l} \frac{1}{2}\left(\frac{\partial v_{k}}{\partial r_{l}}+\frac{\partial v_{l}}{\partial r_{k}}\right),
$$

después considera que, para un sólido isotrópico

$$
\frac{\partial v_{k}}{\partial r_{l}}=\frac{\partial v_{l}}{\partial r_{k}}
$$

sustituyendo la igualdad (B.12) en la ecuación (B.11) resulta

$$
\frac{d \epsilon_{i j}}{d t}=\epsilon_{i k} \frac{1}{2}\left(\frac{\partial v_{j}}{\partial r_{k}}-\frac{\partial v_{k}}{\partial r_{j}}\right)+\epsilon_{k j} \frac{1}{2}\left(\frac{\partial v_{i}}{\partial r_{k}}-\frac{\partial v_{k}}{\partial r_{i}}\right)+\alpha_{i j k l}\left(\frac{\partial v_{k}}{\partial r_{l}}\right) .
$$

Por otra parte en analogía con la ecuación (B.3) escribe la siguiente expresión

$$
\frac{\partial \epsilon_{i j}}{\partial t}=\frac{d \epsilon_{i j}}{d t}-v_{k} \frac{\partial \epsilon_{i j}}{\partial r_{k}}
$$

usando (B.13) y (B.14) escribe finalmente la expresión para la derivada parcial con respecto al tiempo del tensor permitividad

$$
\frac{\partial \epsilon_{i j}}{\partial t}=\frac{1}{2} \epsilon_{i k}\left(\frac{\partial v_{j}}{\partial r_{k}}-\frac{\partial v_{k}}{\partial r_{j}}\right)+\frac{1}{2} \epsilon_{k j}\left(\frac{\partial v_{i}}{\partial r_{k}}-\frac{\partial v_{k}}{\partial r_{i}}\right)+\alpha_{i j k l} \frac{\partial v_{k}}{\partial r_{l}}-v_{k} \frac{\partial \epsilon_{i j}}{\partial r_{k}},
$$

esta es la ecuación buscada, la cual utiliza Robinson como base de su cálculo para obtener la densidad de fuerza en sólidos. 


\section{Apéndice $\mathrm{C}$}

\section{Deducción de las ecuaciones (5.10) y (5.11)}

Para mostrar de donde se obtienen las ecuaciones (5.10) y (5.11) partimos de las siguientes identidades vectoriales y tensoriales

$$
\begin{gathered}
\nabla \cdot(\vec{U} \vec{V})=\vec{V}(\nabla \cdot \vec{U})+(\vec{U} \cdot \nabla) \vec{V} \\
\nabla(\vec{U} \cdot \vec{V})=(\vec{U} \cdot \nabla) \vec{V}+(\vec{V} \cdot \nabla) \vec{U}+\vec{U} \times(\nabla \times \vec{V})+\vec{V} \times(\nabla \times \vec{U}) \\
\vec{U} \times(\nabla \times \vec{V})=(\nabla \vec{V}) \cdot \vec{U}-(\vec{U} \cdot \nabla) \vec{V},
\end{gathered}
$$

de las ecuaciones (C.1), (C.2) y (C.3) podemos escribir

$$
\begin{gathered}
\vec{V}(\nabla \cdot \vec{U})=\nabla \cdot(\vec{U} \vec{V})-(\vec{U} \cdot \nabla) \vec{V} \\
\vec{U} \times(\nabla \times \vec{V})=\nabla(\vec{U} \cdot \vec{V})-(\vec{U} \cdot \nabla) \vec{V}-(\vec{V} \cdot \nabla) \vec{U}-\vec{V} \times(\nabla \times \vec{U}) \\
\vec{V} \times(\nabla \times \vec{U})=(\nabla \vec{U}) \cdot \vec{V}-(\vec{V} \cdot \nabla) \vec{U} .
\end{gathered}
$$

Ahora analizando un vector, que tiene la siguiente forma

$$
\begin{aligned}
\vec{A} & \equiv \vec{V}(\nabla \cdot \vec{U})-\vec{U} \times(\nabla \times \vec{V}) \\
& =\vec{V}(\nabla \cdot \vec{U})-\frac{1}{2} \vec{U} \times(\nabla \times \vec{V})-\frac{1}{2} \vec{U} \times(\nabla \times \vec{V}),
\end{aligned}
$$

sustituyendo las identidades (C.3), (C.4) y (C.5) en la ecuación (C.7)

$$
\begin{aligned}
\vec{A}= & {[\nabla \cdot(\vec{U} \vec{V})-(\vec{U} \cdot \nabla) \vec{V}]-\frac{1}{2}[(\nabla \vec{V}) \cdot \vec{U}-(\vec{U} \cdot \nabla) \vec{V}] } \\
& -\frac{1}{2}[\nabla(\vec{U} \cdot \vec{V})-(\vec{U} \cdot \nabla) \vec{V}-(\vec{V} \cdot \nabla) \vec{U}-\vec{V} \times(\nabla \times \vec{U})],
\end{aligned}
$$

sustituyendo la ecuación (C.6) en la expresión (C.8) resulta

$$
\begin{aligned}
\vec{A}= & {[\nabla \cdot(\vec{U} \vec{V})-(\vec{U} \cdot \nabla) \vec{V}]-\frac{1}{2}[(\nabla \vec{V}) \cdot \vec{U}-(\vec{U} \cdot \nabla) \vec{V}] } \\
& -\frac{1}{2}[\nabla(\vec{U} \cdot \vec{V})-(\vec{U} \cdot \nabla) \vec{V}-(\vec{V} \cdot \nabla) \vec{U}-(\nabla \vec{U}) \cdot \vec{V}+(\vec{V} \cdot \nabla) \vec{U}],
\end{aligned}
$$


C.0.

simplificando y agrupando términos

$$
\vec{A}=\nabla \cdot(\vec{U} \vec{V})-\frac{1}{2} \nabla(\vec{U} \cdot \vec{V})+\frac{1}{2}[(\nabla \vec{U}) \cdot \vec{V}-(\nabla \vec{V}) \cdot \vec{U}]
$$

Usando el hecho de que

$$
\nabla(\vec{U} \cdot \vec{V})=\nabla \cdot \mathbf{I}(\vec{U} \cdot \vec{V})
$$

podemos escribir (C.10) como

$$
\mathbf{A} \equiv \vec{V}(\nabla \cdot \vec{U})-\vec{U} \times(\nabla \times \vec{V})=\nabla \cdot\left[\vec{U} \vec{V}-\frac{1}{2} \mathbf{I}(\vec{U} \cdot \vec{V})\right]+\frac{1}{2}[(\nabla \vec{U}) \cdot \vec{V}-(\nabla \vec{V}) \cdot \vec{U}]
$$

Con este procedimiento hemos mostrado como obtenemos la igualdad tensorial (C.12), que es la base para escribir las ecuaciones (5.10) y (5.11). Para exhibir la forma explícita de estas ecuaciones tenemos que realizar cambios.

Si hacemos el cambio $V \rightarrow \vec{E}$ y $\vec{U} \rightarrow \vec{D}$ en la expresión (C.12) tenemos la siguiente identidad

$$
\vec{E}(\nabla \cdot \vec{D})-\vec{D} \times(\nabla \times \vec{E})=\nabla \cdot\left[\vec{D} \vec{E}-\frac{1}{2}(\vec{D} \cdot \vec{E}) \mathbf{I}\right]+\frac{1}{2}[(\nabla \vec{D}) \cdot \vec{E}-(\nabla \vec{E}) \cdot \vec{D}]
$$

análogamente si cambiamos $V \rightarrow \vec{H}$ y $U \rightarrow \vec{B}$ en la expresión (C.12) obtenemos

$$
\vec{H}(\nabla \cdot \vec{B})-\vec{B} \times(\nabla \times \vec{H})=\nabla \cdot\left[\vec{B} \vec{H}-\frac{1}{2}(\vec{B} \cdot \vec{H}) \mathbf{I}\right]+\frac{1}{2}[(\nabla \vec{B}) \cdot \vec{H}-(\nabla \vec{H}) \cdot \vec{B}] .
$$

Las ecuaciones (C.13) y (C.14) nos permiten mostrar la forma final de nuestras ecuaciones de balance de momento. 


\section{Apéndice D}

\section{Coeficientes del tensor $\alpha_{j k l m}$}

Para deformaciones suficientemente pequeñas se puede escribir

$$
\delta \epsilon_{j k}=\delta S_{l m} \alpha_{j k l m},
$$

donde

$$
\alpha_{j k l m}=\frac{\partial \epsilon_{j k}}{\partial S_{l m}} .
$$

Los 81 coeficientes $\alpha_{j k l m}$ son las componentes de un tensor de cuarto rango. En virtud de las relaciones $\epsilon_{j k}=\epsilon_{k j}, S_{l m}=S_{m l}$, se tiene

$$
\alpha_{j k l m}=\alpha_{k j l m}=\alpha_{j k m l} .
$$

Luego de los 81 - $9=72$ términos no diagonal, sólo el 36 son independientes y el número total de coeficientes independientes se reduce a $36+9=45$. Para reducir aún más el sistema se debe hacer uso de las condiciones de simetría, introduciendo la limitación de es un medio inicialmente isotrópico, aunque no necesariamente homogéneo.

La variación en la densidad de la energía electrostática debido a una pequeña deformación es

$$
\delta u=-\frac{1}{2} a_{j k l m} E_{j} E_{k} \delta S_{l m}
$$

Ahora bien, como el dieléctrico se supone que es inicialmente isotrópico, entonces la ecuación (D.4) debe ser invariante a una inversión en la dirección de cualquier eje coordenado y para el intercambio de cualquiera de los dos ejes de coordenadas. Por lo tanto una inversión del eje $x_{j}$, invierte los signos de $E_{j}$ y $\epsilon_{j k}, k \neq j$, pero deja todo los demás factores sin cambios. Como consecuencia, ciertos coeficientes deben desaparecer si $\delta u$ no es afectada por la reversión o intercambio de ejes. De hecho, es evidente que todos menos tres clases de coeficientes son cero, a saber:

$$
a_{j j j j}=a_{1}, \quad a_{j j k k}=a_{2}, \quad a_{j k j k}=a_{3}, \quad(j \neq k) .
$$

Ahora $\delta u$ también debe ser invariante a la rotación de los ejes de coordenadas y esto implica una relación adicional entre los parámetros $a_{1}, a_{2}$ y $a_{3}$. Usando las 
D.0.

ecuaciones (D.5) en la expresión (D.4) resulta

$$
\begin{aligned}
\delta u= & -\frac{1}{2}\left(a_{1}-a_{2}-4 a_{3}\right) E_{j}^{2} \delta S_{j j}-\frac{1}{2} a_{2} E^{2} \delta S_{j j} \\
& -2 a_{3}\left(E_{j}^{2} \delta S_{j j}+E_{1} E_{2} \delta S_{12}+E_{2} E_{3} \delta S_{23}+E_{3} E_{1} \delta S_{31}\right) .
\end{aligned}
$$

Sólo el primer término de la ecuación (D.6) es invariante con las coordenadas y es necesario que

$$
a_{1}-a_{2}-4 a_{3}=0, \quad a_{3}=\frac{1}{4}\left(a_{1}-a_{2}\right)
$$

Con esto se muestra como el tensor de cuarto rango $\alpha_{j k l m}$, para un sólido tiene unicamente dos constantes independientes, denominadas por Stratton como, $a_{1}$ y $a_{2}$. 
LA ECUACIÓN DE BALANCE DE MOMENTO ELECTROMAGNÉTICO Y LA DENSIDAD DE FUERZA PARA SOLIDOS
En la Ciudad de México, se presentaron a las 15:00 horas del día 20 del mes de octubre del año 2017 en la Unidad Iztapalapa de la Universidad Autónoma Metropolitana, los suscritos miembros del jurado:

DR. VICTOR DAVID GRANADOS GARCIA

DR. ALBERTO RUBIO PONCE

MTRO. JOSE LUIS JIMENEZ RAMIREZ
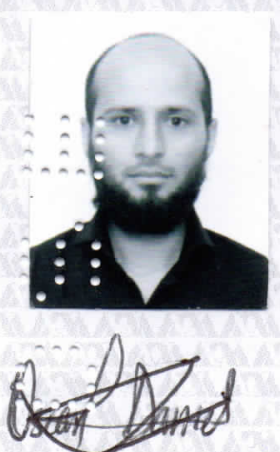

OSCAR DANIEL NUÑEZ VALENCIA ALUMNO

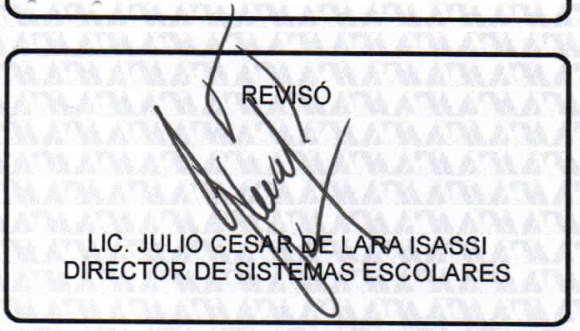

DIRECTOR DE LA DIVISIÓN DE CBI

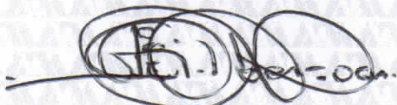

DR. JOSE GILBERTO CORDOBA HERRERA

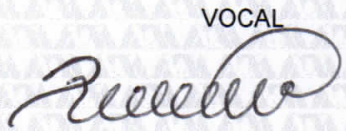

DR. ALBERTO RUBIO PONCE
Bajo la Presidencia del primero y con carácter de Secretario el último, se reunieron para proceder al Examen de Grado cuya denominación aparece al margen, para la obtención del grado de:

MAESTRO EN CIENCIAS (FISICA)

DE: OSCAR DANIEL NUÑEZ VALENCIA

$y$ de acuerdo con el artículo 78 fracción III del Reglamento de Estudios Superiores de la Universidad Autónoma Metropolitana, los miembros del jurado resolvieron:

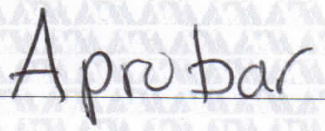

Acto continuo, el presidente del jurado comunicó al interesado el resultado de la evaluación $y$, en caso aprobatorio, le fue tomada la protesta. 